Pacific

Journal of

Mathematics

\title{
HILBERT SPACE REPRESENTATIONS OF THE ANNULAR TEMPERLEY-LIEB ALGEBRA
}

Vaughan F. R. Jones and SaRah A. ReznikofF 


\title{
HILBERT SPACE REPRESENTATIONS OF THE ANNULAR TEMPERLEY-LIEB ALGEBRA
}

\author{
Vaughan F. R. Jones and Sarah A. ReZnikofF
}

\begin{abstract}
The set of diagrams consisting of an annulus with a finite family of curves connecting some points on the boundary to each other defines a category in which a contractible closed curve counts for a certain complex number $\delta$. For $\delta=2 \cos (\pi / n)$ this category admits a $C^{*}$-structure and we determine all Hilbert space representations of this category for these values, at least in the case where the number of internal boundary points is even. This result has applications to subfactors and planar algebras.
\end{abstract}

\section{Introduction}

The annular Temperley-Lieb algebra ATL has a parameter $\delta$ and is linearly spanned by isotopy classes of $(m, n)$ diagrams. For $m$ and $n$ nonnegative integers, an $(m, n)$ diagram consists of an annulus with $m$ marked points on the inside circle and $n$ marked points on the outside connected to each other by a family of smooth disjoint curves, called strings, inside the annulus. There may also be (necessarily closed) curves that do not connect boundary points. If such a curve is homologically trivial in the annulus, the diagram may be replaced by the same one with the closed curve removed, but multiplied in the algebra by $\delta$. By definition a basis of ATL consists of such diagrams with no homologically trivial circles. Multiplication of an $(m, p)$ diagram $T$ by a $(p, n)$ diagram $S$ is achieved by identifying the outside boundary $T$ with the inside boundary of $S$ in such a way that the boundary points coincide, smoothing the strings at the $p$ common marked boundary points, and removing the common boundary to produce the annular diagram $S T$.

To the best of our knowledge, the first explicit investigation of ATL appeared in [Jones 1994], where it was encountered in a concrete form as an algebra of linear transformations on the tensor powers of the $n \times n$ matrices (and $\delta=n^{2}$ ). This study was relatively simple because of the concrete situation and the fact that as soon as

MSC2000: primary 46L37; secondary 16D60, 57M27.

Keywords: planar algebras, subfactors, annular Temperley-Lieb, category, affine Hecke.

Jones' research was supported in part by NSF Grants DMS 9322675 and 0401734 and the Marsden fund UOA520. This research was conducted in part while Reznikoff was a postdoctoral fellow with John Phillips at the University of Victoria and supported by NSERC of Canada, and in part while she was a visiting assistant professor at Reed College. 
$n$ is greater than 2 the algebra is "generic" and the structure of the representations does not depend on $n$. Also, homologically nontrivial circles in the annulus are no different from contractible ones, which, as we shall see, is quite special.

The second explicit analysis of ATL appeared in [Graham and Lehrer 1998], where the abstract algebra more or less as defined in the first paragraph was defined and studied in its own right. One can no longer avoid homologically nontrivial strings, and the version of ATL in that article introduced a second parameter to account for this. Graham and Lehrer produced an impressively complete analysis and we have been greatly inspired by their results. In [Jones 2001] we showed how to use ATL to obtain results about subfactors. It was recognized that, for a general planar algebra $P$, the operadic concept of a module over $P$ is the same thing as an ordinary module over a canonically defined algebra spanned by annular tangles in $P$. This led to the perhaps confusing notion of "TL-module" in [Jones 2001], which in fact means an ordinary module over the annular algebra. Here we work with a generalization of ATL, which permits shadings on tangles of either parity. The affine TL algebroid AffTL with parameter $\delta \in \mathbb{C}$ is the category with objects the elements of $\mathbb{N} \times\{+,-\}$, and morphisms from $(m, \operatorname{sgn})$ to $\left(n, \operatorname{sgn}^{\prime}\right)$ the elements of the vector space $\operatorname{AffTL}_{(m, s g n),\left(n, \mathrm{sgn}^{\prime}\right)}$ having as basis the set of shaded affine $(2 m, 2 n)$-diagrams. Multiplication between composable morphisms is the linear extension of the map on basis elements given by $\beta \alpha=\delta^{c(\beta \circ \alpha)}(\beta \circ \alpha)$. A convention determines how a diagram is shaded according to the sign in its index.

A representation of AffTL is a covariant functor from this category into the category of vector spaces. Applications to subfactors require that we restrict attention to representations on vector spaces with positive-definite and AffTL-invariant natural sesquilinear forms - these are the "Hilbert" modules. The main result of this paper is a complete characterization of the irreducible Hilbert AffTL modules.

In analogy with the situation for the ordinary Temperley-Lieb algebra, the natural candidates for the irreducible modules of AffTL are the quotients of

$$
\operatorname{AffTL}_{\left(k, \operatorname{sgn}^{\prime}\right),(n, \operatorname{sgn})}
$$

by diagrams with fewer than $k$ through strings (for $k \in 2 \mathbb{N}$ ). It makes sense to restrict to $\operatorname{sgn}^{\prime}=+$, and in this annular situation we also need to introduce a parameter $\omega$ to correspond to the effect of rotation on the internal annulus boundary, and quotient by this relation as well. (In the case $k=0$, which is slightly different but no more complicated, factors of $\omega$ correspond instead to pairs of homologically nontrivial curves in the annulus.) The resulting vector spaces are denoted $V_{n, \operatorname{sgn}}^{k, \omega}$. It turns out that every irreducible representation of the algebroid is isomorphic to a quotient of some $V_{n, \text { sgn }}^{k, \omega}$; on the other hand, if the natural sesquilinear form on $V_{n, \mathrm{sgn}}^{k, \omega}$ is positive semidefinite, then in fact its quotient by the length zero vectors is an irreducible representation (denoted $\mathscr{V}_{n}^{k, \omega}$ ). 
In [Jones 2001] it was shown that in the generic case the form is actually positive definite on $V_{n, \text { sgn }}^{k, \omega}$. Here we determine the exact set of parameter values corresponding to the irreducibles in the nongeneric case. The main result (for the case $k$ positive; again, $k=0$ is similar) is that when $\delta=2$ or $2 \cos \frac{\pi}{a}$, then $\mathscr{V}^{k, \omega}$ exists if and only if $\delta=2$ and $\omega=1$, or $\omega=q^{2 r}$ where $r$ is such that $k<r \leq a / 2$ and $q+q^{-1}=\delta$. As a corollary to the proof, we obtain the generating functions for the dimensions of the irreducible modules in terms of Tchebychev polynomials.

The first main ingredient in our proof, which was used in the paper just mentioned, is the observation that $\mathscr{V}_{n}^{k, \omega}$ can be viewed as an ordinary Temperley-Lieb module, and thus decomposed into a direct sum of the irreducible modules of this algebra. Positive definiteness of the sesquilinear form is checked on these summands individually. Checking the form on the copy of the trivial TemperleyLieb representation, which is the image of the Jones-Wenzl idempotent, is the difficult part. A formula of Graham and Lehrer [1998] settles this question at each level $n$ of the module; one of the main components of our paper is a reworking of their result to suit our context. There are some simple nongeneric cases in which this formula alone serves to prove or disprove the existence of $\mathscr{V}_{n}^{k, \omega}$, but for the general case, once $n$ is large enough so that the abstract Temperley-Lieb algebra is no longer semisimple we need to pass to a quotient of $V_{n}^{k, \omega}$ before we can use the formula. Once we obtain the suitable quotient, the inductive proof of positivity on the summands goes through, and determining when the trivial representation is present can be done as usual with the Graham-Lehrer formula.

The paper is structured as follows. In Chapter 2 we establish notation and recall the relevant background material concerning the ordinary Temperley-Lieb algebra. Chapter 3 introduces the affine algebra and the family of vector spaces $V_{n}^{k, \omega}$. Chapter 4 is devoted to Graham and Lehrer's theorem. In Chapter 5 we state and prove a necessary condition on $\omega$ for $\mathcal{V}_{n}^{k, \omega}$ to exist in the nongeneric case; namely, that $\omega=e^{\frac{\pi i}{a}}$ for some integer $r$ with $k<r \leq a / 2$, where $\delta=2 \cos \frac{\pi}{a}$. In Chapter 6 it is shown that this condition is in fact sufficient, and thus, together with the results of [Jones 2001] (along with a brief analysis of the case $\delta=2$ ), it completely characterizes the nongeneric irreducible Hilbert space representations of the algebra AffTL.

\section{Notation}

We use the notation $[n]=\frac{q^{n}-q^{-n}}{q-q^{-1}}$ throughout this paper.

The Tchebychev polynomials $P_{n}(x)=[n]$ with $x=q+q^{-1}$ satisfy $P_{n+1}=$ $x P_{n}(x)-P_{n-1}(x)$ and we define essentially the same polynomials $Q_{n}(z)$ by $Q_{0}=0$, $Q_{1}=1$ and $Q_{n+1}(z)=Q_{n}(z)-z Q_{n-1}(z)$. Note that $x^{n-1} Q_{n}\left(x^{-2}\right)=[n]$. 
For the entirety of this paper, if $\mu$ is a complex number we let $\omega$ be such that $\mu=\sqrt{\omega}+\sqrt{\omega}^{-1}$. We have $\omega=-1$ if and only if $\mu=0$.

It will be important to distinguish clearly between the abstract Temperley-Lieb algebra defined by multiplication on a basis of diagrams, and a quotient of it which supports a $C^{*}$-algebra structure, and is only defined for special values of the parameter. So let $\mathrm{TL}_{m}(\delta)$ be the ${ }^{*}$-algebra over $\mathbb{C}$ with basis formed by systems of disjoint curves (called strings) in a rectangle with $m$ boundary points on the top and bottom as usual, with multiplication of diagrams $\alpha$ and $\beta$ defined by stacking $\alpha$ on top of $\beta$ and removing closed strings with a multiplicative factor $\delta$. See [Kauffman 1987] and [Jones 1999] for details. (The $*$ structure is defined by reflecting diagrams in a straight line half way between the bottom and top of a rectangle.)

It is important to note that there is a natural inclusion of $\mathrm{TL}_{n}$ in $\mathrm{TL}_{n+1}$, obtained by adding a new through string to the right of a basis element of $\mathrm{TL}_{n}$. We will often make the identification of $\mathrm{TL}_{n}$ with a subalgebra of $\mathrm{TL}_{n+1}$ without comment.

Here is a picture of an element $E_{i}$ in $\mathrm{TL}_{n}$, where $i=1, \ldots, n-1$ :

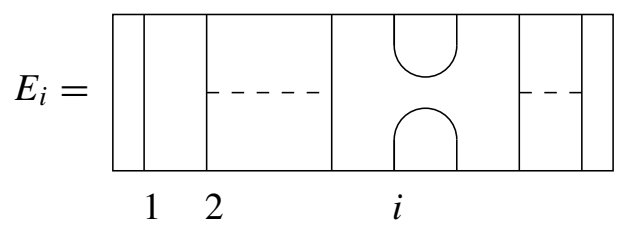

In [Jones 1983], for $\delta \geq 2$ and $\delta=2 \cos \pi / a$ for each integer $a=3,4,5, \ldots$ we constructed a tower of $C^{*}$-algebras, which we will call $\mathscr{T} \mathscr{L}_{n}$ for $n=1,2,3, \ldots$ generated by the identity and orthogonal projections $e_{i}, i=1,2, \ldots, n-1$, which satisfy the relations $e_{i} e_{i \pm 1} e_{i}=\delta^{-2} e_{i}$ and $e_{i} e_{j}=e_{j} e_{i}$ for $|i-j| \geq 2$. It is well known (see [Goodman et al. 1989]) that there is a $*$-algebra homomorphism $\Phi_{n}$ from $\mathrm{TL}_{n}$ onto $\mathcal{T} \mathscr{L}_{n}$ sending $E_{i}$ onto $\delta e_{i}$. This homomorphism is compatible with the inclusions of $\mathrm{TL}_{n} \subseteq \mathrm{TL}_{n+1}$ and $\mathscr{T} \mathscr{L}_{n} \subseteq \mathscr{T} \mathscr{L}_{n+1}$. $\Phi$ is "generically" (i.e. for $\delta \geq 2$ ) an isomorphism. When $\Phi$ is not an isomorphism it is known (see below) that its kernel is the ideal generated by the "Jones-Wenzl" (JW) idempotent $p_{n} \in \mathrm{TL}_{n}$ defined in [Wenzl 1987] by the inductive formula

$$
p_{1}=1, \quad p_{n+1}=p_{n}-\frac{[n]}{[n+1]} p_{n} E_{n} p_{n}
$$

with $\delta=q+q^{-1}$ as long as $[j] \neq 0$ for $j=1,2, \ldots, n+1$.

The unique irreducible representation of $\mathrm{TL}_{n}$ on which $E_{1}$ (hence all $E_{i}$ ) acts by zero will be called the trivial representation. Note that this passes to $\mathscr{T} \mathscr{L}_{n}$ exactly when $n<a-1$. 
For the convenience of the reader we give a proof that the kernel of $\Phi$ is the ideal generated by the JW idempotent. Our proof will actually give a set of basis elements of $\mathrm{TL}_{n}$ that span a subalgebra mapped isomorphically onto $\mathscr{T} \mathscr{L}_{n}$ by $\Phi$.

Theorem 2.1 (Goodman-Wenzl). The kernel of the map $\Phi: \mathrm{TL}_{n} \rightarrow \mathscr{T} \mathscr{L}_{n}$ is the ideal generated by $p_{a-1}$ for $n \geq a-1$.

Proof. First we construct a sequence $A_{n}$ of subalgebras of $\mathrm{TL}_{n}$. For $n<a-1$ let $A_{n}=\mathrm{TL}_{n}$ and proceed inductively, setting $A_{n+1}=A_{n} E_{n} A_{n}$ for $n \geq a-2$. (Clearly $A_{n}$ has a basis consisting of words on the $E_{i}$ 's.) Although the $A_{n}$ are not included in one another, each is individually an algebra. To see this use the maps ("conditional expectations") $\mathscr{E}_{n}: \mathrm{TL}_{n+1} \rightarrow \mathrm{TL}_{n}$ defined on the diagram basis by connecting the rightmost top and bottom boundary points of a $\mathrm{TL}_{n+1}$ diagram to give a $\mathrm{TL}_{n}$ diagram. It is clear that $\mathscr{E}_{n}\left(x E_{n} y\right)=x y$ for $x, y \in \mathrm{TL}_{n}$ and that $E_{n} x E_{n}=\mathscr{E}_{n-1}(x) E_{n}$ for $x \in \mathrm{TL}_{n}$.

One then proves inductively the following three assertions:

(i) $A_{n}$ is a subalgebra of $\mathrm{TL}_{n}$.

(ii) $\mathscr{E}_{n}\left(A_{n+1}\right) \subseteq A_{n}$.

(iii) $A_{n}$ is an $A_{n-1}-A_{n-1}$ bimodule under multiplication in $\mathrm{TL}_{n}$.

Now let $I_{n}$ be the ideal in $\mathrm{TL}_{n}$ generated by the JW idempotent $p_{a-1}$ defined above. Observe that $I_{n} \subseteq I_{n+1}$. It follows immediately from the standard form of words on the $E_{i}$ 's (see e.g. [Jones 1983]) that $\mathrm{TL}_{n+1}=\left(\mathrm{TL}_{n}\right) E_{n}\left(\mathrm{TL}_{n}\right) \oplus \mathbb{C}$ id for all $n$. So since $1-p_{a-1} \in\left(\mathrm{TL}_{n}\right) E_{n}\left(\mathrm{TL}_{n}\right)$ for $n \geq a-1$, we have $\mathrm{TL}_{n+1}=$ $\left(\mathrm{TL}_{n}\right) E_{n}\left(\mathrm{TL}_{n}\right) \bmod \left(I_{n+1}\right)$ for $n \geq a-1$. Thus by induction

$$
\mathrm{TL}_{n+1}=A_{n} E_{n} A_{n} \quad \bmod \left(I_{n+1}\right) \quad \text { for } n \geq a-1 .
$$

We now show, also by induction, that $\left.\Phi\right|_{A_{n}}$ is an isomorphism onto $\mathscr{T} \mathscr{L}_{n}$. This assertion for $n=a-1$ is in some sense the main point of [Jones 1983] since $\operatorname{ker}\left(\Phi_{a-1}\right)$ is spanned by $1-p_{a-1}$. Now consider the commutative diagram

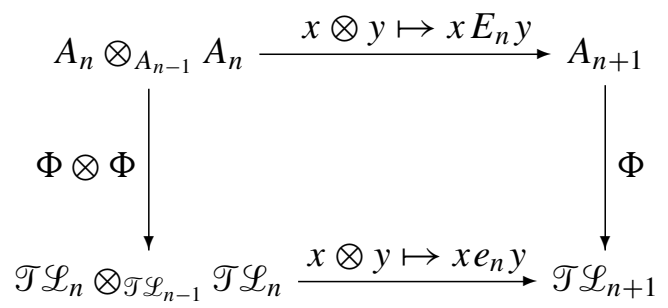

All the maps in this diagram are $A$-bimodule homomorphisms where $\mathscr{T} \mathscr{L}$ becomes an $A$ - $A$ bimodule by transport of structure. It is shown in [Goodman et al. 1989] that the bottom horizontal arrow is an isomorphism. The top horizontal arrow is surjective. It follows that the restriction of $\Phi_{n+1}$ to $A_{n+1}$ is an isomorphism.

Together with $(*)$, this proves the theorem. 
Thus the tower of algebras $\mathscr{T} \mathscr{L}_{n}$ admits a Bratteli diagram, which was shown in [Jones 1983] to be of the form below (exhibited for $\delta=2 \cos \pi / 7$ ).

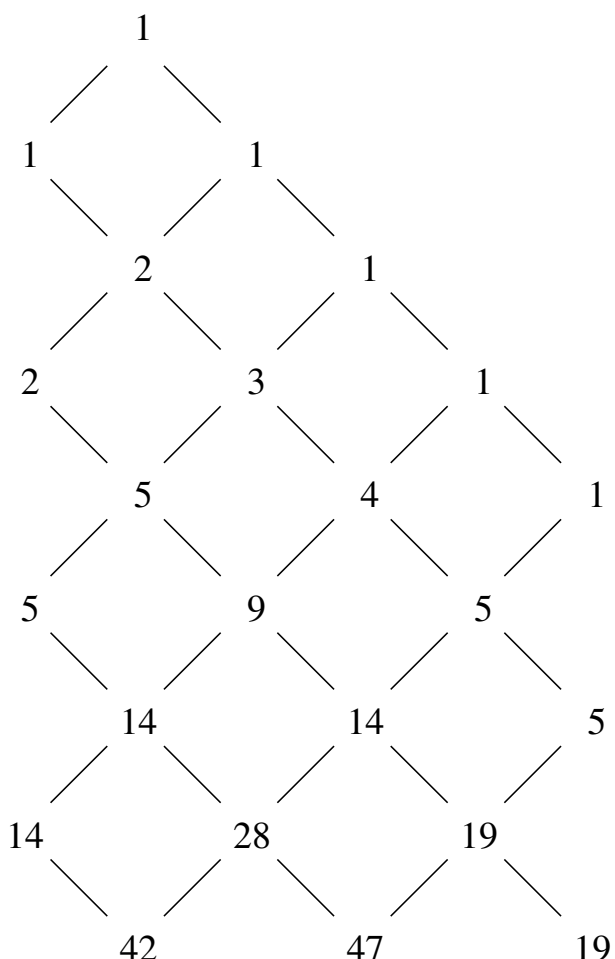

This Bratteli diagram can alternatively be thought of as giving the Hilbert space representations of TL, which may be obtained explicitly as follows. For each $n=$ $0,1,2, \ldots$ and each $t \leq n$ with $t \equiv n \bmod 2$, a $(t, n)$ planar diagram is defined to be a rectangle with $n$ marked points on the top and $t$ on the bottom joined pairwise by disjoint smooth curves inside the rectangle. A curve is a through string if it connects the bottom to the top of the rectangle. $W_{n}^{t}$ is defined to be the vector space whose basis is the set of $(t, n)$ planar diagrams with $t$ through strings. $\mathrm{TL}_{n}$ acts on $W_{n}^{t}$ by concatenation of diagrams (as multiplication is defined in $\mathrm{TL}_{n}$ itself), except that the result is zero if there are fewer than $t$ through strings in the concatenated diagram. There is an invariant inner product $\langle\cdot, \cdot\rangle$ on $W_{n}^{t}$ defined by $\langle\alpha, \beta\rangle=\beta^{*} \alpha$, which is an element of the one dimensional vector space $W_{t}^{t}$. This inner product is positive semidefinite and the quotient $W_{n}^{t}$ is a Hilbert space affording a representation of $\mathscr{T} \mathscr{L}_{n}$. This result is well known to the experts but probably does not appear anywhere in the literature.

Since the source of positivity for our annular Temperley-Lieb modules will be that of this inner product, we give a reasonably detailed proof here - tracking positivity down to its von Neumann algebraic origin. 
Theorem 2.2. For $\delta \geq 2$ or $\delta=2 \cos \pi / a$, for $a=3,4,5, \ldots$, the inner product on $W_{n}^{t}$ defined in the previous paragraph is positive semidefinite; that is, $\langle\alpha, \alpha\rangle \geq 0$ for all $\alpha$.

Proof. We will effectively identify the representation on $\mathcal{W}_{n}^{t}$ with the action on a principal left ideal in $\mathscr{T} \mathscr{L}_{n}$, which has a positive definite inner product coming from the Markov trace of [Jones 1983].

The algebra $\mathscr{T} \mathscr{L}_{n}$ was analyzed in [Jones 1983] using the basic construction. Adopting that technique, by induction, the irreducible representations $\psi_{t}$ for $0 \leq$ $t \leq a-2$ with $t \equiv n \bmod 2$ so obtained are uniquely defined up to equivalence by the following property: if $p$ is the largest integer such that $\psi_{t}\left(e_{1} e_{3} e_{5} \ldots e_{2 p-1}\right)$ is not zero, then $p=\frac{1}{2}(n-t)$. For each such $t$ let $q_{t}$ be the minimal central idempotent in $\mathscr{T} \mathscr{L}_{n}$ corresponding to $\psi_{t}$ and define another inner product $\{\cdot, \cdot\}$ on $W_{n}^{t}$ by

$$
\{\alpha, \beta\}=\operatorname{tr}\left(\Phi\left(\tilde{\beta}^{*} \tilde{\alpha}\right) q_{t}\right)
$$

where tr denotes the Markov trace of [Jones 1983] and, given a basis diagram $\gamma \in W_{n}^{t}$, we write $\tilde{\gamma}$ for the $\mathrm{TL}_{n, n}$ diagram obtained from $\gamma$ in the following fashion:

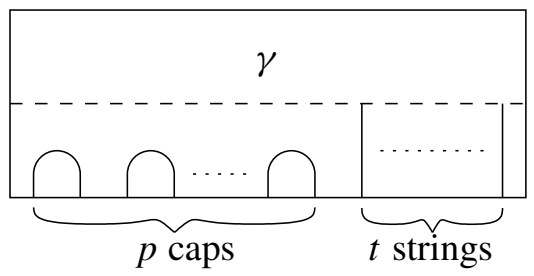

Now observe that if $\beta^{*} \alpha$ has fewer than $t$ through strings then $\Phi\left(\tilde{\beta}^{*} \tilde{\alpha}\right) q_{t}=0$. This is because $\tilde{\beta}^{*} \tilde{\alpha}$ may be written in the form $\gamma_{1} E_{1} E_{3} E_{5} \ldots E_{2 k-1} \gamma_{2}$ with $k>$ $\frac{1}{2}(n-t)$. On the other hand if $\beta^{*} \alpha$ has $t$ through strings then

$$
\tilde{\beta}^{*} \tilde{\alpha}=\langle\alpha, \beta\rangle \Phi\left(E_{1} E_{3} E_{5} \ldots E_{2 p-1}\right) .
$$

Thus in this case the Markov trace of $\left(\tilde{\beta}^{*} \tilde{\alpha}\right) q_{t}$ is a positive multiple, $K$, depending only on $n, \delta$, and $t$, of $\langle\alpha, \beta\rangle$. Combining the two possibilities for the number of through strings we see that in any case there is a $K \geq 0$ such that $\{\alpha, \beta\}=K\langle\alpha, \beta\rangle$. Since the trace on a $\mathrm{II}_{1}$ factor gives a positive definite inner product, $\{\cdot, \cdot\}$ is positive semidefinite and so is $\langle\cdot, \cdot\rangle$.

We shall now obtain formulae for the dimensions of the individual $\mathscr{W}_{n}^{t}$ for $\delta=$ $2 \cos \pi / a$. To this end let $d_{t, m}=\operatorname{dim}\left(W_{t+2 m}^{t}\right)$ for $t=0,1,2, \ldots, a-2$ and $m=$ $0,1,2, \ldots$ Then the meaning of the Bratteli diagram is precisely that

$$
d_{t, m}=d_{t-1, m}+d_{t+1, m-1},
$$


with $d_{t,-1}=0$ for all $t, d_{a-1, n}=0$ for all $n$ and $d_{-1,0}=1$ but $d_{-1, n}=0$ for $n>0$. By induction these relations uniquely determine the $d_{t, n}$. If we form the generating functions

$$
D_{t}(z)=\sum_{n=0}^{\infty} d_{t, n} z^{n}
$$

then these relations are equivalent to

$$
z D_{t+1}=D_{t}-D_{t-1}
$$

with $D_{a-1}=0$ and $D_{-1}=1$.

Thus any power series $D_{t}(z)$ satisfying these conditions must be the generating functions for the $d_{t, n}$. But if $Q_{r}$ are the modified Tchebychev polynomials defined above then setting $D_{t}(z)=Q_{a-t-1}(z) / Q_{a}(z)$ we see that the relations are satisfied.

We see we have proved the following.

Theorem 2.3. For $\delta=2 \cos \pi / a$ and all integers $t \geq 0$, the generating function $D_{t}(z)=\sum_{n=0}^{\infty} \operatorname{dim} \mathcal{W}_{t+2 n}^{t} z^{n}$ is equal to $Q_{a-t-1} / Q_{a}$.

Remark 2.4. The ordinary Temperley-Lieb algebras may be turned into an algebroid in the obvious way with objects being the nonnegative integers and morphisms from $m$ to $n$ being linear combinations rectangular Temperley-Lieb diagrams with $m$ points on the bottom boundary and $n$ on the top. (So the morphisms are the zero vector space if $m$ and $n$ are different modulo 2.) It is clear that if we define for each $t$ the vector space (graded by $m$ ), $\mathcal{W}^{t}=\left\{\mathcal{W}_{m}^{t}\right\}$ to be zero if $t<m$ or $t$ and $m$ are not equal modulo 2, then these are the Hilbert space modules over the algebroid.

\section{Affine Temperley-Lieb}

Motivated by a conjecture of Freedman and Walker, we are going to define a slightly different version of the annular Temperley-Lieb algebra from that of [Jones 2001]. It will be essentially the same as that of [Graham and Lehrer 1998]. The difference is in how isotopies are required to act on the boundary. In order to avoid confusion with the definitions of [Jones 2001], we will here call our diagrams "affine" rather than annular.

In the following definition, for a positive integer $k,\{k\}$ will denote the set of $k$-th roots of unity in $\mathbb{C}$. "The" annulus $A$ will mean the set of complex numbers $z$ with $1 \leq|z| \leq 2$.

Definition 3.1. Let $m$ and $n$ be two nonnegative integers equal mod 2. An affine $(m, n)$ TL diagram is the intersection with the annulus of a system of smooth closed curves (strings) in $\mathbb{C}$ that meet the boundary of the annulus transversally, precisely 
in the points $\{m\}$ and $2\{n\}$. Such diagrams are considered to be the same if they differ by isotopies of the annulus which are the identity on the boundary.

An affine TL diagram is called connected if it has no closed curves in the interior of the annulus.

A through string in an affine TL diagram is a string whose end points lie on different boundary components of $A$.

To make the set of all affine TL diagrams into a category we compose an $(m, p)$ diagram $\alpha$ with a $(p, n)$ diagram $\beta$ by $\beta \circ \alpha=O(2 \beta \cup \alpha)$, where we have smoothed the strings of $\alpha$ and $2 \beta$ where they meet and $O$ is the transformation of $\mathbb{C}$ which sends $r e^{i \theta}$ to $\sqrt{r} e^{i \theta}$. (Smoothing could be avoided by requiring the isotopies to be the identity in a neighborhood of the boundary and insisting that the strings be $C^{\infty}$ perpendicular to the boundary.)

If $m$ and $n$ are even, an affine TL diagram admits a shading, that is, a 2-coloring of the connected components of the complement of the strings in $A$, so that two components whose closures meet have different colors. The precise category that will interest us is the category with two objects $(n, \pm)$ for each nonnegative integer $n$ and where the set of morphisms from $(m, \pm)$ to $(n, \pm)$ is the set of shaded affine $(2 m, 2 n)$ TL diagrams. Shadings are determined by the following convention where + means shaded and - means unshaded: if $\beta$ is a diagram giving a morphism from $(m, \operatorname{sgn})$ to $\left(n, \operatorname{sgn}^{\prime}\right)$ then on the inner boundary of $A$ a small region close to 1 and in the first quadrant is shaded according to sgn and a small region close to 2 and in the first quadrant is shaded according to $\operatorname{sgn}^{\prime}$. We illustrate this here by giving an example of a morphism from $(2,-)$ to $(3,+)$.

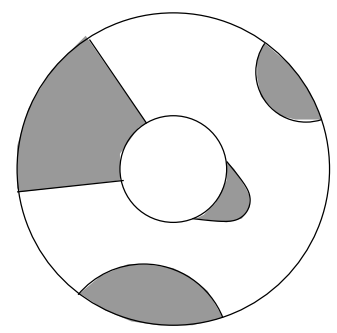

Given an affine TL diagram $\alpha, \hat{\alpha}$ will denote the connected diagram formed by removing all contractible closed strings from $\alpha$, and $c(\alpha)$ will be the number of contractible closed strings in $\alpha$.

Definition 3.2. The affine TL algebroid AffTL with parameter $\delta \in \mathbb{C}$ will be the category with objects the elements of $\mathbb{N} \cup\{0\} \times\{+,-\}$, and where the set of morphisms from $(m, \pm)$ to $(n, \pm)$, denoted $\operatorname{AffTL}_{(m, \pm),(n, \pm)}$, is the vector space having as basis the set of shaded connected affine TL $(2 m, 2 n)$ diagrams as above, with multiplication between composable morphisms defined to be the linear extension 
of the map on basis elements given by

$$
\beta \alpha=\delta^{c(\beta \circ \alpha)} \widehat{\beta \circ \alpha} .
$$

A representation of AffTL will be a covariant functor from this category into the category of vector spaces.

The transformation $z \mapsto 2 / \bar{z}$ of $\mathbb{C}$ preserves affine TL diagrams and so defines a conjugate-linear antiinvolution * of the algebroid AffTL. A representation $\pi$ of AffTL will be called a Hilbert representation if the representing vector spaces are Hilbert spaces and $\pi\left(\alpha^{*}\right)=\pi(\alpha)^{*}$ for all diagrams $\alpha$.

Remark 3.3. Having taken linear combinations of annular diagrams we can now give a meaning to an annular diagram which also contains a (contractible) rectangle with $2 m$ boundary points labeled by an element $x \in \mathrm{TL}_{m}$. Such a diagram will mean the linear combination of annular diagrams obtained by writing $x$ as a linear combination of basis elements and inserting those basis elements in the rectangle to obtain a linear combination of AffTL elements. The beginning boundary point on the rectangle would need to be marked if there were any ambiguity.

Hilbert representations admit an obvious direct sum operation and in this paper we wish to classify all Hilbert representations into the category of finite dimensional Hilbert spaces. They will all be quotients of a universal family which we now define.

For the rest of this section we suppose that sgn is a fixed sign, + or - , and all statements are to be true for both values of sgn.

Definition 3.4. For any positive integer $k$ and complex number $\omega$ let $V_{n, \text { sgn }}^{k, \omega}$ be the graded vector space (graded by the subscripts, in $(\mathbb{N} \cup\{0\}) \times\{+,-\})$ that is the quotient of $\operatorname{AffTL}_{(k,+),(n, \text { sgn })}$ by the subspace spanned by all diagrams with fewer than $2 k$ through strings (so that $V_{n, \text { sgn }}^{k, \omega}=0$ for $n<k$ ) and all elements of the form $\alpha \rho-\omega \alpha$, where $\rho \in \operatorname{AffTL}_{(k,+),(k,+)}$ is the diagram all of whose strings are through strings and for which 1 is connected to $2 e^{4 \pi i / 2 k}$. (We will use the notation $\rho_{k}$ if we need to specify the actual number of strings $\rho$ has. Note that $\rho_{k}^{k}$ is the rotation by $2 \pi$.)

For $\omega \neq-1$ and $\mu \neq \delta$ we let $V_{n \text {,sgn }}^{0, \omega}$ be the graded vector space (graded by $(\mathbb{N} \cup\{0\}) \times\{+,-\})$ that is the quotient of the vector space $\operatorname{AffTL}_{(0,+),(n, \text { sgn })}$ by the linear span of elements of the form $\alpha \sigma^{*} \sigma-\bar{\mu} \mu \alpha$, where $\sigma$ is the diagram in $\operatorname{AffTL}_{(0,+),(0,-)}$ having exactly one closed homologically nontrivial (in $A$ ) string.

For $k=0$ and $\mu=\delta$ we let $V_{n, \text { sgn }}^{0, \omega}$ be the vector space with basis the set of all ordinary Temperley-Lieb diagrams in a disc with boundary points being the $2 n$-th roots of unity, and having the shading determined by sgn. This is acted on in the obvious way by AffTL. Note also that it is the quotient of $\operatorname{AffTL}_{(0, \operatorname{sgn}),(n, \mathrm{sgn})}$ by 
the relation that sets a diagram equal to any other diagram with the same system of connections between boundary points.

For $\omega=-1$ (hence $\mu=0$ ) we let

(a) $V_{n, \operatorname{sgn}}^{0,-1, \text { sgn }}$ be the quotient of the vector space $\operatorname{AffTL}_{(0, \mathrm{sgn}),(n, \mathrm{sgn})}$ by the linear span of elements of the form $\alpha \sigma^{*} \sigma$, and

(b) $V_{n, \mathrm{sgn}}^{0,-1,-\mathrm{sgn}}$ be the quotient of the vector space $\operatorname{AffTL}_{(0,-\mathrm{sgn}),(n, \mathrm{sgn})}$ by the linear span of elements of the form $\alpha \sigma^{*}$ (or $\alpha \sigma$ according to sgn).

Remark 3.5. Note that $V_{n, \mathrm{sgn}}^{0, \omega}$ depends on $\mu$ only through $\omega\left(\right.$ as $\left.\mu=\sqrt{\omega}+\sqrt{\omega}^{-1}\right)$, so using $\omega$ in the notation is justified.

The special treatment of the case $\omega=-1$ is unfortunate but unavoidable. If one defined two different such representations in all cases, then if $k \neq 0$ they would be isomorphic via either $\rho$ or a diagram with one homologically nontrivial circle, but this last map is not invertible if $\mu=0$. Also of course these two representations $V^{0,-1, \pm}$ are inequivalent since the two spaces graded by 0 have different dimensions.

Remark 3.6. Since composition of tangles does not increase the number of through strings and the action of tangles on the inside annular boundary commutes with the action on the outside, the $V^{k, \omega}$ become modules over AffTL by composition in that category.

Remark 3.7. Observe that $V_{n, \pm}^{k, \omega}$ is finite dimensional for fixed $k$ and $n$. We will need their dimensions, which can be calculated by counting diagrams exactly as in [Jones 2001]:

(a) For $k>0$ and $n \geq k, \operatorname{dim} V_{n, \pm}^{k, \omega}=\left(\begin{array}{c}2 n \\ n-k\end{array}\right)$.

(b) For $k=0, \mu=0$ and $n>0$, we have $\operatorname{dim} V_{n, \pm}^{0, \omega, \pm}=\frac{1}{2}\left(\begin{array}{c}2 n \\ n\end{array}\right), \operatorname{dim} V_{0, \text { sgn }}^{0,-1, \operatorname{sgn}}=1$, and $\operatorname{dim} V_{0,-\operatorname{sgn}}^{0,-1, \operatorname{sgn}}=0$.

(c) For $k=0$ and $\mu=\delta, \operatorname{dim} V_{n, \pm}^{0, \omega}=\frac{1}{n+1}\left(\begin{array}{c}2 n \\ n\end{array}\right)$.

(d) For $k=0$ and $0<\mu<\delta, \operatorname{dim} V_{n, \pm}^{0, \omega}=\left(\begin{array}{c}2 n \\ n\end{array}\right)$.

For uniformity of notation, in the case $k=0, \mu=0$ we will use the superscript $\omega$ to denote the pair $(-1, \pm)$ in the above formulae.

We now define the key ingredient of this paper, a sesquilinear form on each $V_{n, \pm}^{k, \omega}$. To this end note that the quotient $\operatorname{Aff}_{k, \text { sgn }}$ of $\operatorname{AffTL}_{(k, \operatorname{sgn}),(k, \text { sgn })}$ by the subspace spanned by diagrams with fewer than $k$ through strings is a unital $*$-algebra freely generated by the element $\rho$ when $k>0$, and $\sigma^{*} \sigma$ when $k=0$. These generators 
are unitary and self-adjoint, respectively. Thus if $|\omega|=1$ and $\mu \in \mathbb{C}$ we may define unital $*$-algebra homomorphisms $\phi: \operatorname{Aff}_{k, \text { sgn }} \rightarrow \mathbb{C}$ by $\phi(\rho)=\omega$, and $\phi\left(\sigma^{*} \sigma\right)=$ $\bar{\mu} \mu$ respectively. We also use the letter $\phi$ for the ${ }^{*}$-algebra homomorphism from $\operatorname{AffTL}_{(k, \mathrm{sgn}),(k, \text { sgn })}$ to $\mathbb{C}$ obtained by composing with the quotient map.

Definition 3.8. With notation as in the last paragraph, define the sesquilinear forms $\langle\cdot, \cdot\rangle$ on each $\operatorname{AffTL}_{(k,+),(n, \text { sgn })}$ by $\langle v, w\rangle=\phi\left(w^{*} v\right)$.

Proposition 3.9. The sesquilinear form of Definition 3.8 is invariant; that is, $\langle\alpha v, w\rangle=\left\langle v, \alpha^{*} w\right\rangle$.

Proof. This follows immediately from $w^{*} \alpha v=(\alpha w)^{*} v$ and the fact that $\phi$ is a *-algebra homomorphism.

Proposition 3.10. The sesquilinear form of Definition 3.8 passes to the quotient $V_{n, \mathrm{sgn}}^{k, \omega}$.

Proof. If $\delta \neq \mu$ it follows from the $*$-homomorphism property of $\phi$ that the elements defined in Definition 3.4 spanning the subspace by which the quotient was taken are orthogonal to all diagrams in $\operatorname{AffTL}_{(k,+),(n, \mathrm{sgn})}$. Case (b) of the definition requires care. One observes that if $v=\alpha \sigma$ and $w$ is any diagram in the same space then $w^{*} v$ is actually a multiple of $\delta$ times an element of the form $\beta \sigma^{*} \sigma$. This is because, after the removal of homologically trivial circles, $w^{*} v$ has a homologically nontrivial circle, hence at least two because the shadings near the inner and outer boundaries have to match.

Finally in the case $\mu=\delta$, if two diagrams $v$ and $v^{\prime}$ define the same system of connections among boundary points, the diagrams for $w^{*} v$ and $w^{*} v^{\prime}$ are both the same system of closed curves with the inner annulus boundary possibly in different regions. The homologically nontrivial closed curves must occur in pairs for the annulus boundary shadings to match, and since $\mu=\delta$, such a pair will count the same if it is dealt with by $\phi$ or if it is homologically trivial.

The element $1 \in V_{k,+}^{k, \omega}$ clearly generates $V^{k, \omega}$ as a representation of AffTL. We will call it the vacuum vector and write it $v_{\omega}$. It satisfies the following properties, where the $\epsilon_{i}$ are as in Definition 2.8 of [Jones 2001].

(a) When $k>0$, we have $\left\langle v_{\omega}, v_{\omega}\right\rangle=1, \rho\left(v_{\omega}\right)=\omega v_{\omega}$, and $\epsilon_{i}\left(v_{\omega}\right)=0$ for $0 \leq i \leq$ $2 k-1$.

(b) When $k>0$, we have $\left\langle v_{\omega}, v_{\omega}\right\rangle=1$ and $\sigma^{*} \sigma\left(v_{\omega}\right)=\bar{\mu} \mu v_{\omega}$.

The following fundamental lemma was poorly treated in [Jones 2001]. This was because the conclusion was obvious from spherical invariance in the planar algebras to which it was applied. We give a careful proof here.

Lemma 3.11. The inner products in $V^{k, \omega}$ can be calculated using just properties (a) and (b) above. 
Proof. Since any vector in $V^{k, \omega}$ is a linear combination of affine diagrams applied to $v_{\omega}$, it suffices by invariance to show how the equations can be used to calculate $\left\langle\alpha v_{\omega}, v_{\omega}\right\rangle$, with $\alpha$ connected. First consider the case $k>0$. Then $\alpha$ is a connected affine tangle with $2 k$ inner and outer boundary points. If all the strings are not through strings, $\alpha=\alpha^{\prime} \epsilon_{i}$ for some $i$, and the inner product is zero. If all the strings are through strings $\alpha$ is necessarily some power of $\rho$ so the inner product is determined by properties $\left\langle v_{\omega}, v_{\omega}\right\rangle=1$ and $\rho\left(v_{\omega}\right)=\omega v_{\omega}$.

Now suppose $k=0$. Then by connectedness $\alpha$ consists of a certain number of strings which may be isotoped into concentric circles. They must be even in number since the inner and outer boundaries have the same shading. This means precisely that $\alpha$ is a power of $\sigma^{*} \sigma$.

Corollary 3.12. Any Hilbert representation of AffTL is isomorphic to a quotient of $V^{k, \omega}$ for some root of unity $\omega$, and the corresponding $\langle\cdot, \cdot\rangle$ of Definition 3.8 is positive semidefinite. If $k=0$, then $0 \leq \mu \leq \delta$.

Proof. As in [Jones 2001], if $\mathcal{U}$ is an irreducible Hilbert space representation, all the $U_{m, \text { sgn }}$ have to be irreducible $\operatorname{AffTL}_{(m, s g n),(m, \mathrm{sgn})}$ modules. Let $k$ be the smallest integer for which $\boldsymbol{U}_{k, \pm}$ is nonzero (this $k$ is called the lowest weight and $\boldsymbol{U}_{(k, \pm)}$ is called the lowest weight space). Then $\operatorname{AffTL}_{(k, \text { sgn }),(k, s g n)}$ acts on the lowest weight space via the abelian quotient $\mathrm{AffTL}_{k}$,sgn defined before Definition 3.8. By some version of Schur's lemma the lowest weight space is thus one dimensional and the unitary $\rho$ must act by some $\omega$, with $|\omega|=1$, or, if $k=0, \sigma^{*} \sigma$ must act by some nonnegative real — choose $\mu$ to be a nonnegative square root of that constant and then choose $\omega$ accordingly. A lowest weight vector of unit length in $u_{k,+}$ will then satisfy all the conditions of (a) or (b) above so we may define a $\langle\cdot, \cdot\rangle$-preserving map from the corresponding $V^{t, \omega}$ onto $U$ by sending, for any connected $\alpha, \alpha\left(v_{\omega}\right)$ onto the $\alpha$ applied to a lowest weight unit vector $u \in U$. So $\langle\cdot, \cdot\rangle$ is positive semidefinite, since the inner product in $U$ is.

The case $k=0, \omega=-1$ does not quite work as above. Then either $u_{0,+}$ or $u_{0,-}$ must be nonzero; suppose that it is $U_{0 \operatorname{sgn}}$ and choose $u$ therein. Then $\|\sigma(u)\|=0$ (or $\left\|\sigma^{*}(u)\right\|=0$ ), so by irreducibility $u_{0, \text { sgn }}$ vanishes. Then proceed as before to obtain an isomorphism between $U$ and $V^{0,-1 \text { sgn }}$.

That $\mu \leq \delta$ follows as in [Jones 2001].

Conversely, if $\langle\cdot, \cdot\rangle$ is positive semidefinite on some $V^{k, \omega}$, the quotient by its kernel is a Hilbert space representation of AffTL, which we call $\mathscr{q}^{k, \omega}$.

Lemma 3.13. $\mathscr{q}^{k, \omega}$ is irreducible.

Proof. Suppose $v \in \mathscr{V}^{k, \omega}$ is nonzero. Then $\langle v, v\rangle \neq 0$. But $v=\sum_{\alpha} c_{\alpha} \alpha v_{\omega}$ for some affine diagrams $\alpha$ and constants $c_{\alpha}$. So $\left\langle\sum_{\alpha} \overline{c_{\alpha}} \alpha^{*} v, v_{\omega}\right\rangle \neq 0$; since AffTL $_{k s g n}$ is one-dimensional it follows that $v_{\omega}$, hence all of $\mathscr{V}^{k, \omega}$, is in the AffTL span of $v$. 
Definition 3.14. If $U$ is an irreducible representation of AffTL isomorphic to $q^{k, \omega}$ then $k$ will be called the lowest weight of $U$ and $\omega$ will be called the chirality.

The determination of the set of values of $\delta, \omega$ and $\mu$ for which the sesquilinear form $\langle\cdot, \cdot\rangle$ is positive semidefinite is the subject of the next sections.

Finally remark that the representations $V^{k, \omega}$ are all mutually inequivalent except when $k=0$ (when clearly $V^{k, \omega}$ and $V^{k, \omega^{-1}}$ are the same). Also $V^{0,0,+}$ and $V^{0,0,-}$ are inequivalent since the dimensions of the spaces graded by $(0,+)$ and $(0,-)$ are different. Thus at the end of this paper we will have obtained a complete list of irreducible Hilbert representations of AffTL.

\section{The formula of Graham and Lehrer}

Let $V^{k, \omega}$, with $|\omega|=1$ or $0 \leq \mu \leq \delta$, be the affine Temperley-Lieb module constructed in the previous section and let $v_{\omega}$ be a lowest weight unit vector therein.

Definition 4.1. For $k \leq n$ we will call $\alpha_{n}$ the element of $\operatorname{AffTL}_{(k,+),(n,+)}$ containing one copy of the JW idempotent $p_{2 n}$ in a rectangle whose first boundary point is connected to -2 and the next $2 n-1$ (in cyclic order) are also connected to the outside boundary of $A$. The $2 k$ boundary points on the inside boundary of $A$ are connected to the middle $2 k$ of the remaining boundary points of the rectangle and the other boundary points of the rectangle are connected to each other in the unique (planar) way so that none is connected to its nearest neighbor.

We illustrate this definition in Figure 1 for $k=2$ and $n=5$. The boundary points of the inner circle are the fourth roots of unity and the ones on the outer circle are the tenth $(=2 n$-th) roots of unity. The order of shaded regions on the boundary

Figure 1

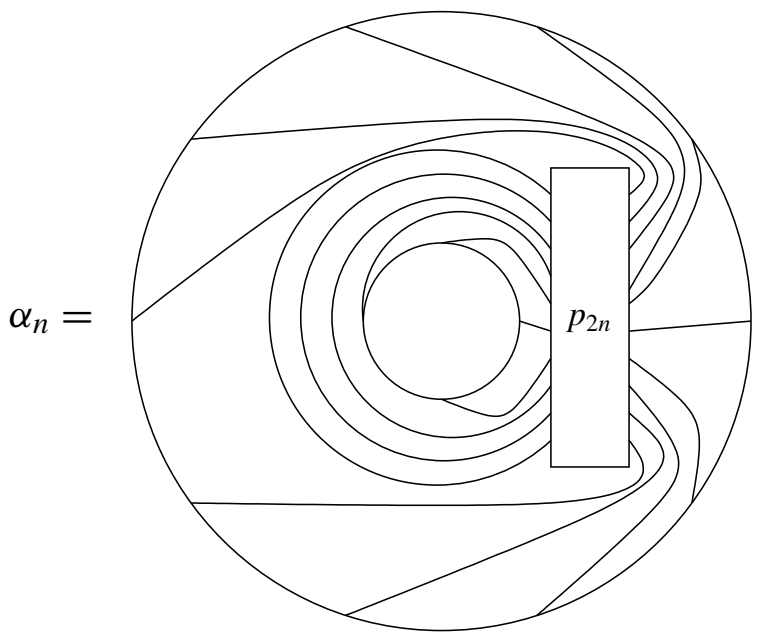


of the rectangle containing $p_{2 n}$ will depend on the parity of $n$, but the ordinary TL algebra makes sense without shading the regions.

Note that Figure 1 represents a (nonzero) linear combination of annular $(2,6)$ tangles obtained by expanding the J-W idempotent $p_{2 n}$ in the rectangle.

For each $r \geq 0$ we set $2 n=2 k+2 r$ and define the vector $w_{n} \in V_{n}^{k, \omega}$ to be the result of applying the annular element $\alpha_{n}$ of Figure 1 to $v_{\omega}$.

Let $C_{n}=\left\langle w_{n}, w_{n}\right\rangle$ for $n>k$ and $C_{k}=1$. Our main task in this paper will be to establish whether $C_{n}$ is positive, negative or zero.

Theorem 4.2. Suppose $\delta\left(=q+q^{-1}\right)$ and $n$ satisfy $\delta>2 \cos \pi / 2 n \geq 0$ (so that in particular the map $\Phi: \mathrm{TL}_{2 n-1} \rightarrow \mathscr{T}_{2 n-1}$ is an isomorphism, and the JW idempotent $p_{2 n}$ is defined). Then with $r=n-k$,

$$
C_{n}=\frac{[r][r+k]}{[2 n][2 n-1]}\left(q^{2 n}+q^{-2 n}-\omega-\omega^{-1}\right) C_{n-1}
$$

Proof. First note that neither [2n] nor [2n-1] is zero. If $\delta>2$ this is obvious. Otherwise write $\delta=2 \cos \pi / a$ ( $a$ not necessarily an integer) so that the condition becomes $\pi / 2 n>\pi / a$, or $2 n \pi / a<\pi$. Then $[2 n]=\sin (2 n \pi / a) / \sin (\pi / a)$, which is strictly positive.

We want to calculate $C_{n}=\left\langle\alpha_{n}\left(v_{\omega}\right), \alpha_{n}\left(v_{\omega}\right)\right\rangle$. By invariance it will suffice to express $\alpha_{n}^{*} \alpha_{n}$ in terms of $\alpha_{n-1}^{*} \alpha_{n-1}$, which we proceed to do.

Case (i), $k>0$.

In Figure 2 we have drawn $\alpha_{n}^{*} \alpha_{n}$ (with $n=4$ for clarity, rather than 5 as in the previous figure).

The first step is to introduce a JW idempotent on one less string. Because of the order on these idempotents, the AffTL element in Figure 3 is the same as in Figure 2 .

Figure 2

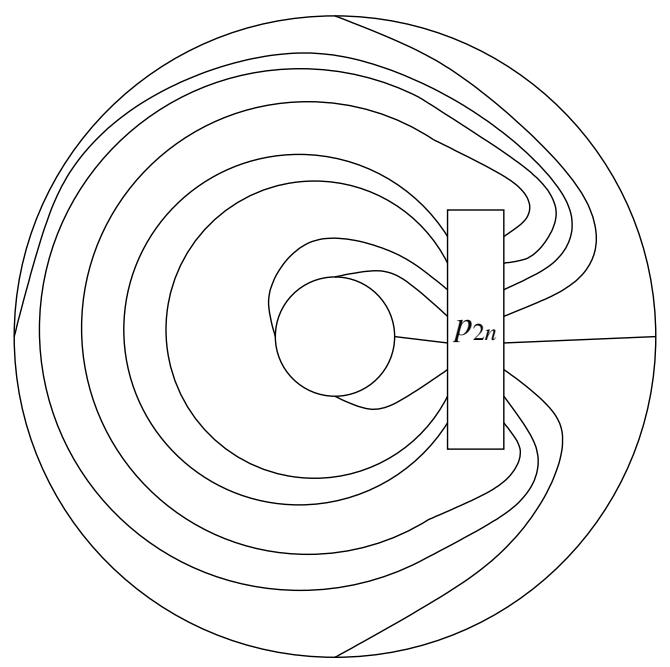




\section{Figure 3}

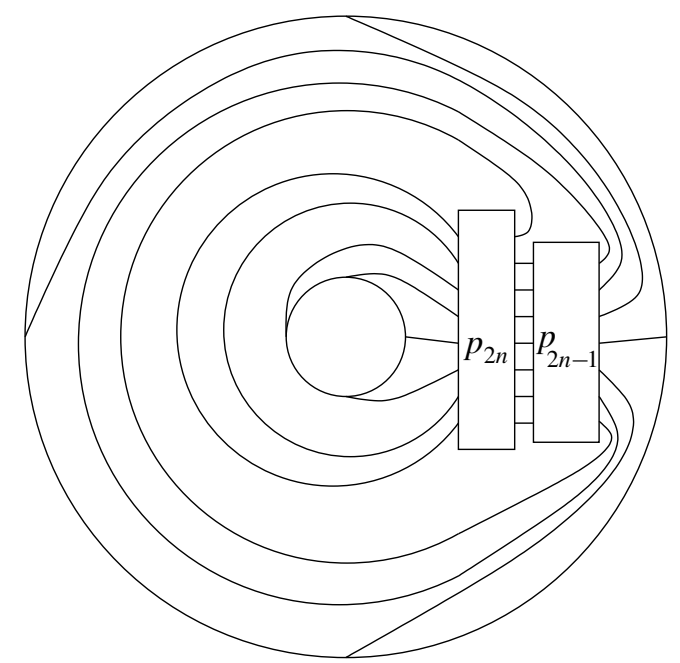

It is easily seen that there are only 3 tangles in the expansion of $p_{2 m}$ that give nonzero contributions in Figure 3 . There must be at least $2 n-2$ through strings inside the rectangle, or two adjacent boundary points on the left side of the rectangle containing $p_{2 n-1}$ would be connected - giving zero. So the only adjacent boundary points on the right side of the $p_{2 n}$ rectangle that can be connected are the top two. Then it is easy to check that the only two pairs of adjacent boundary points on the left side of the $p_{2 n}$ rectangle that can be connected are the ones having exactly one point connected to the inner boundary circle of $A$. Thus we see that

$$
\alpha_{n}^{*} \alpha_{n}=X+c_{Y} Y+c_{Z} Z
$$

where $X, Y$ and $Z$ are given in Figures 4, 5, and 6, respectively.

Figure 4

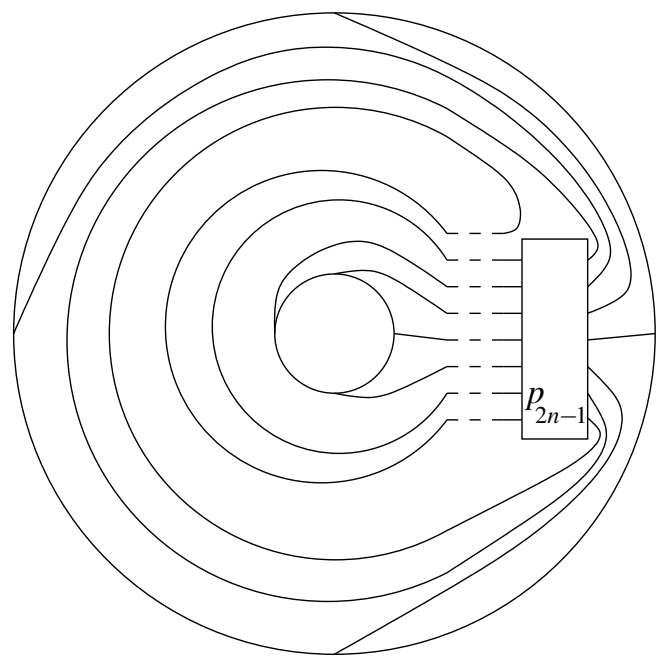




\section{Figure 5}

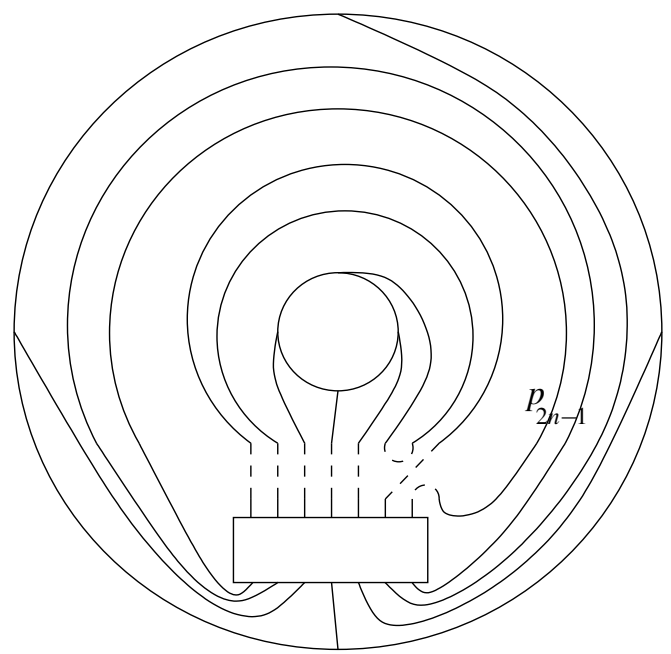

Figure 6

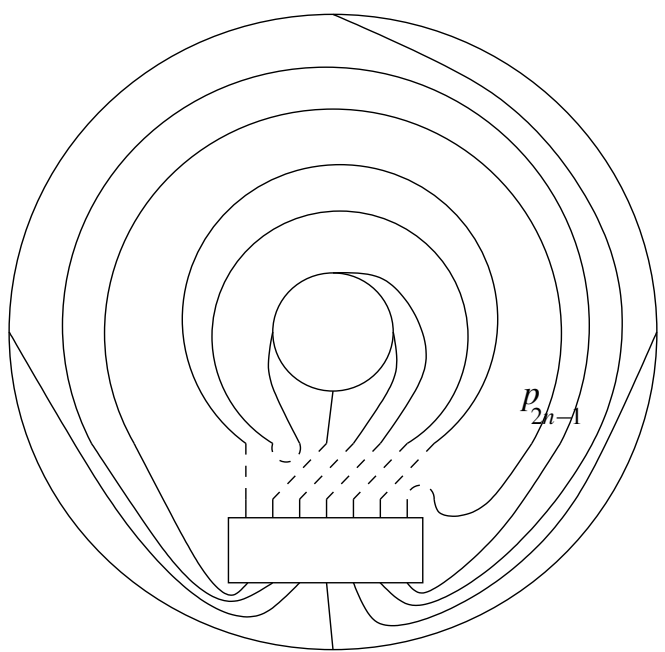

We deal first with the situation in Figure 4. Here after an isotopy we see that the bottom left and right boundary points of the $p_{2 n-1}$ rectangle are connected to each other. The result is well known to be a multiple of $p_{2 n-2}$. By comparing the coefficient of the identity in the expansion of the idempotent, the multiple is seen to be $[2 n] /[2 n-1]$, so that

$$
X=([2 n] /[2 n-1]) \alpha_{n-1}^{*} \alpha_{n-1} .
$$

The arguments for $Y$ and $Z$ are structurally identical and differ only in the constants and the direction in which the inner circle is rotated. In Figure 5, the diagram inside the rectangle for $p_{2 n}$ is $E_{r} E_{r-1} \cdots E_{1}$, which has a coefficient of 
$(-1)^{r}[r+2 k] /[2 n]$ in JW; see [Jones 2001], for example. In Figure 6, the diagram is $E_{r+2 k} E_{r+2 k-1} E_{r+2 k-2} \cdots E_{1}$; this diagram's coefficient is $(-1)^{r}[r] /[2 n]$. Since we will be doing many of these calculations, we record the relevant coefficient in the JW idempotent pictorially:

Figure 7

$r-1$

Coefficient in JW of

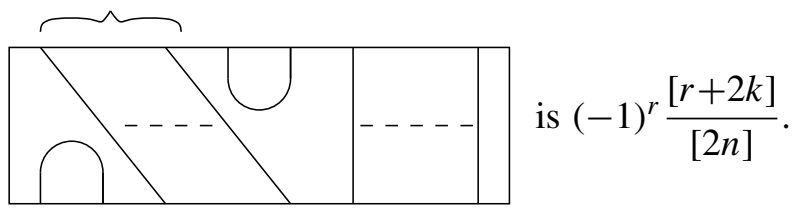

Thus at this stage we have

$$
\alpha_{n}^{*} \alpha_{n}=\frac{[2 n]}{[2 n-1]} \alpha_{n-1}^{*} \alpha_{n-1}+(-1)^{r}\left(\frac{[r+2 k]}{[2 n]} Y+\frac{[r]}{[2 n]} Z\right) .
$$

If we now start with Figure 6 and insert a $p_{2 n-2}$ we obtain Figure 8.

\section{Figure 8}

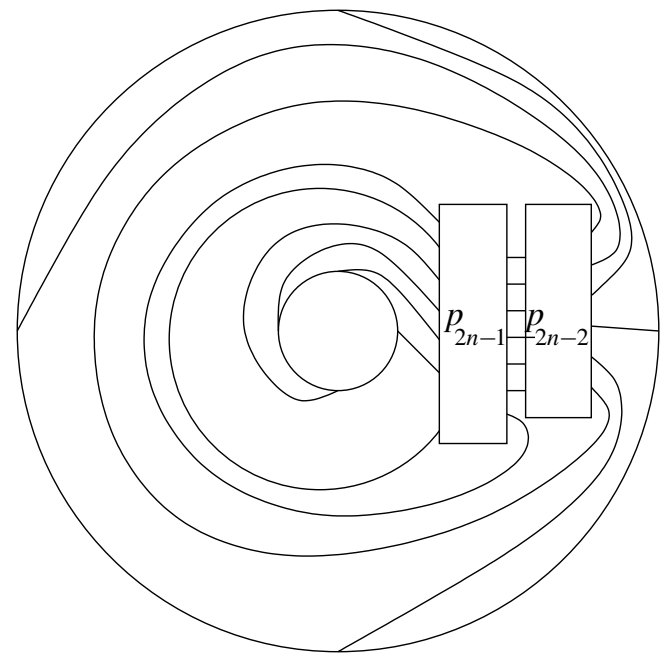

For $Z$, consideration of all possible TL diagrams inside the $p_{2 n-1}$ rectangle shows that the only ones with a nonzero contribution are those with $2 n-2$ through strings and the top and bottom boundary points of the inner annulus boundary connected down and up respectively to their nearest neighbors, as in Figures 9 and 10.

The coefficient of the TL diagram from Figure 9 is, again by Figure 7,

$$
\frac{(-1)^{r+1}[r+2 k]}{[2 n-1]}
$$

and the coefficient of the TL diagram from Figure 10 is

$$
\frac{(-1)^{r+1}[r]}{[2 n-1]}
$$


Figure 9

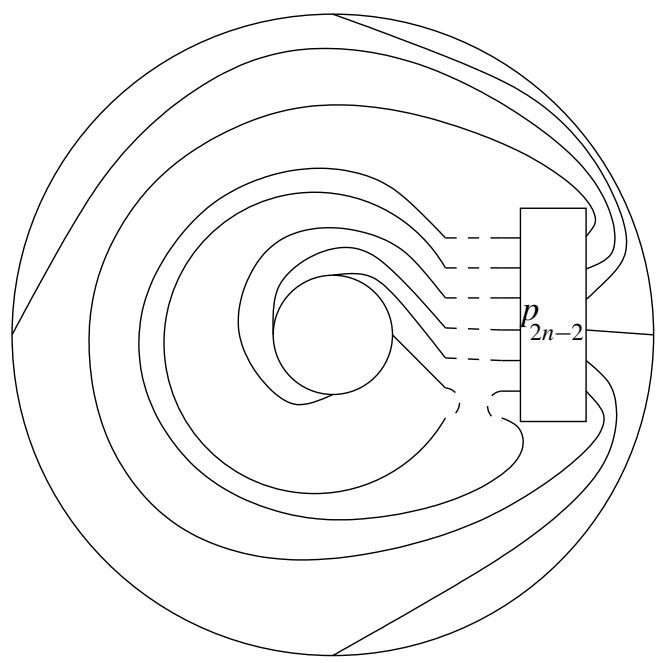

Figure 10

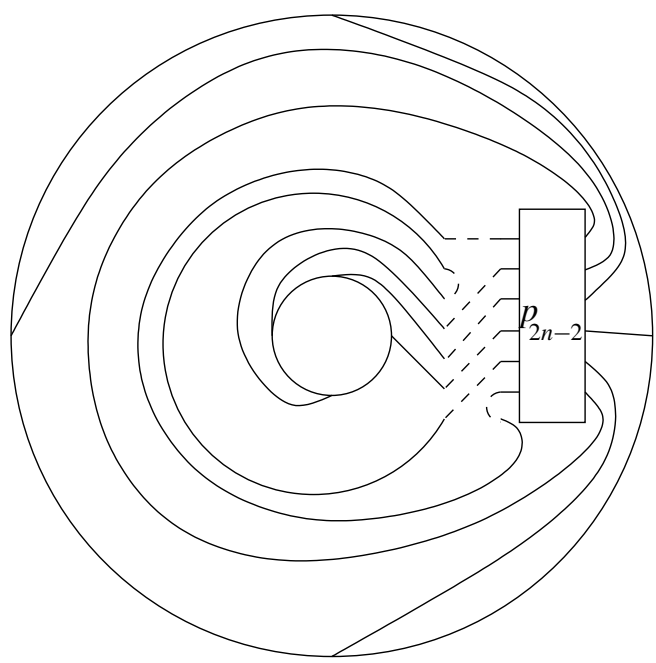

But notice that Figure 9 is just $\alpha_{n-1}^{*} \alpha_{n-1}$ composed with $\rho$ and Figure 10 is just $\alpha_{n-1}^{*} \alpha_{n-1}$. So we have

$$
Z=(-1)^{r+1} \alpha_{n-1}^{*} \alpha_{n-1}\left(\frac{[r+2 k]}{[2 n-1]} \rho+\frac{[r]}{[2 n-1]}\right) .
$$

Doing the corresponding calculation for Figure 5 we obtain that

$$
Y=(-1)^{r+1} \alpha_{n-1}^{*} \alpha_{n-1}\left(\frac{[r+2 k]}{[2 n-1]}+\frac{[r]}{[2 n-1]} \rho^{-1}\right) .
$$


So $C_{Y} Y+C_{Z} Z$ equals

$$
\frac{-1}{[2 n][2 n-1]} \alpha_{n-1}^{*} \alpha_{n-1}\left([r]^{2}+[2 k+r]^{2}-[r][r+2 k]\left(\rho+\rho^{-1}\right)\right) .
$$

Altogether,

$$
\alpha_{n}^{*} \alpha_{n}=\frac{[r][r+2 k]}{[2 n][2 n-1]} \alpha_{n-1}^{*} \alpha_{n-1}\left(\frac{[2 n]^{2}}{[r][r+2 k]}-\frac{[r]}{[r+2 k]}-\frac{[r+2 k]}{[r]}-\rho-\rho^{-1}\right) .
$$

But we have the identity $[2 n]^{2}-[r]^{2}-[2 n-r]^{2}=\left(q^{2 n}+q^{-2 n}\right)[r][2 n-r]$, and on $V_{k}^{k, \omega}, \rho=\omega$ so that

$$
C_{n}=\frac{[n-k][n+k]}{[2 n][2 n-1]}\left(q^{2 n}+q^{-2 n}-\omega-\omega^{-1}\right) C_{n-1}
$$

This proves the theorem when $k>0$.

The case $\mu=\delta$ needs no consideration since in this case ordinary TL diagrams inside a disc provide a Hilbert representation.

Case (ii); $0<\mu<\delta$.

We may consider Figure 3 when $k=0$. In this case there are only two ways to fill in the $p_{2 n}$ rectangle to obtain nonzero diagrams. The first is with the identity which gives $\frac{[2 n]}{[2 n-1]}$ as before, and the second, which is the common case $r=n$ of the terms $Y$ and $Z$ in the previous argument, so we have:

$$
\alpha_{n}^{*} \alpha_{n}=\frac{[2 n]}{[2 n-1]} \alpha_{n-1}^{*} \alpha_{n-1}+(-1)^{n} \frac{[n]}{[2 n]} Y^{\prime}
$$

where $Y^{\prime}$ is the tangle with the inner annulus boundary surrounded by a homologically nontrivial circle as illustrated in Figure 11.

Introducing a $p_{2 n-2}$ as before there is only one contributing diagram that can be put in the $p_{2 n-1}$ rectangle and its coefficient is

$$
\frac{(-1)^{n-1}[n]}{[2 n-1]} \text {. }
$$

The resulting annular diagram is Figure 12.

Note that the innermost circle is an annulus boundary and the next two are strings, which contribute precisely $\sigma^{*} \sigma$. Thus we have

$$
\alpha_{n}^{*} \alpha_{n}=\alpha_{n-1}^{*} \alpha_{n-1}\left(\frac{[2 n]}{[2 n-1]}-\frac{[n]^{2}}{[2 n][2 n-1]} \sigma^{*} \sigma\right) .
$$

But $\sigma^{*} \sigma$ acts as $\mu^{2}$ on $V_{0}^{0, \omega}$ so that

$$
C_{n}=\left(\frac{[2 n]}{[2 n-1]}-\frac{\mu^{2}[n]^{2}}{[2 n][2 n-1]}\right) C_{n-1} .
$$


Figure 11

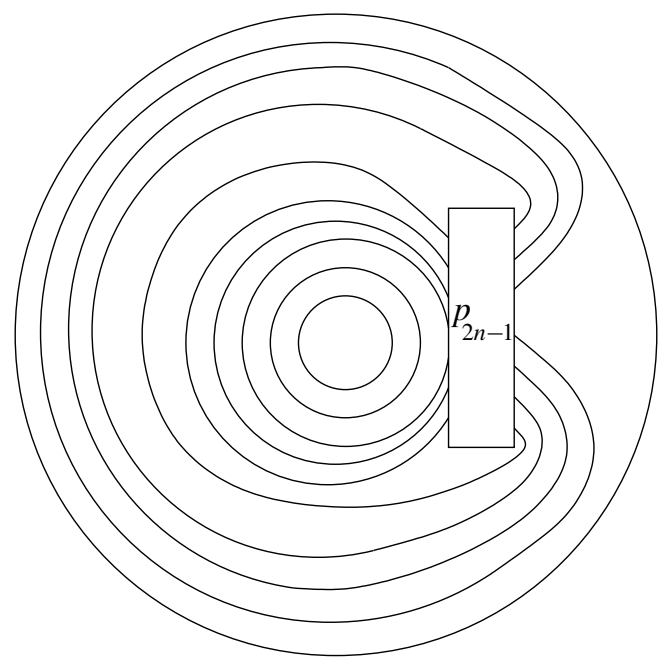

Figure 12

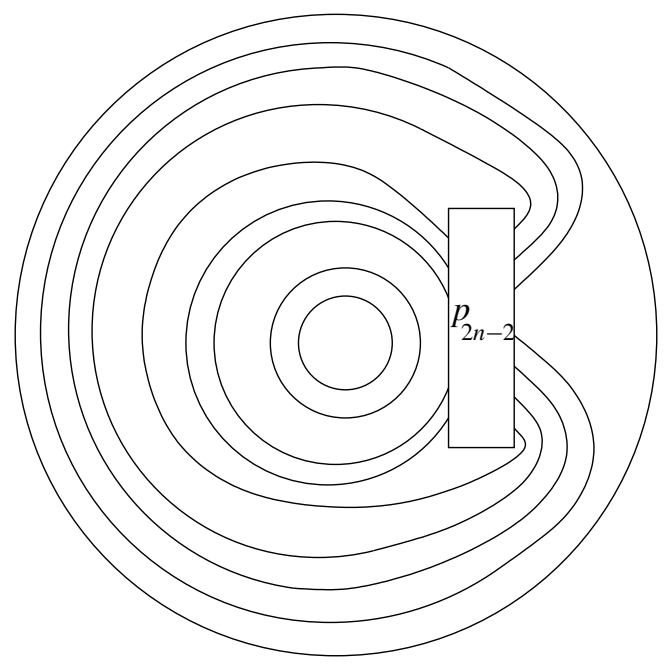

Using $[m]=\frac{q^{m}-q^{-m}}{q-q^{-1}}$ and $\mu^{2}=2+\omega+\omega^{-1}$ we get

$$
C_{n}=\frac{[n]^{2}}{[2 n][2 n-1]}\left(q^{2 n}+q^{-2 n}-\omega-\omega^{-1}\right) C_{n-1} .
$$

This proves the theorem in case (ii).

The only remaining case is $k=0, \omega=0$, where of course $\omega+\omega^{-1}$ is taken to mean zero. In this case the argument is extremely simple as the term $Y$ in case (ii) already acts by zero. Note that in fact there are two cases for $w_{n}$ in this situation according to the shading on the inside annulus boundary. This does not change the argument in any way. 


\section{Restrictions on $\delta, k$ and $\omega$ when $\delta<2$}

Theorem 5.1. Suppose $U$ is an irreducible Hilbert space AffTL module with lowest weight $k$ and chirality $\omega$. Suppose $\delta=2 \cos \pi / a$ for $a=3,4, \ldots$ (choose $\left.q=e^{\pi i / a}\right)$. Then

$$
\omega=q^{ \pm 2 r} \quad \text { for some integer } \quad r \quad \text { with } \quad k<r \leq a / 2 .
$$

Proof. Our first job is to show that $k<a / 2$. Suppose $2 k \geq a$ and let $u$ be a unit vector spanning $\bigcup_{k}$. Then consider the $a$ annular tangles $v_{l}, l=1, \ldots, a$ with $2 k$ inner boundary points and $2 k+2$ outer ones, $2 k$ through strings, with $2 e^{l \pi i /(k+1)}$ connected to $2 e^{(l+1) \pi i /(k+1)}$ and 1 connected to 2 . The matrix of inner products of the vectors $v_{l}(u)$ is the $a \times a$ matrix with $\delta$ on the diagonal, one on the first off-diagonals and 0 elsewhere. The determinant of this matrix is well known to be

$$
[a+1]=\frac{\sin (a+1) \pi / a}{\sin \pi / a},
$$

which is negative. This is impossible in a Hilbert space, so $k<a / 2$.

Since the rotation is unitary and $0 \leq \mu \leq \delta$ we know that $|\omega|=1$ and we may suppose, by taking the complex conjugate if necessary, that $\operatorname{Im}(\omega) \geq 0$. Let $\theta=\arg (\omega)$. We know from Corollary 3.12 that $U$ is a quotient of a $V^{k, \omega}$ so that it makes sense to talk about $v_{k}, w_{n}$ etc. Then if $\theta$ is not $2 r \pi / a$ for some $r$ with $k<r \leq a / 2$ then let $r_{0}$ be the largest value of $r$ with $\theta \geq 2 r \pi / a$. Suppose first that $2\left(r_{0}+1\right)<a$. Then by Theorem 4.2 we have $C_{m}>0$ for $k \leq m \leq r_{0}$ but $C_{m}<0$ for $m=r_{0}+1$, which is disallowed by positivity. So we may suppose $2 r_{0}<a$ but $2\left(r_{0}+1\right) \geq a$. We will divide the proof into two cases.

Case (i) $a$ odd so that $2 r_{0}+1=a$.

The difficulty is clear: using Theorem 4.2 we get $C_{m}>0$ for $1 \leq m \leq r_{0}$, but we cannot apply the theorem for $r_{0}+1$ since its hypotheses are no longer valid.

But the vector $w_{r_{0}}$ still exists and by Theorem 4.2 it is nonzero. Now form, as above, $2 r_{0}+1$ vectors in $u_{r_{0}+1}$ from $w_{r_{0}}$ by applying $2 r_{0}+1$ annular $\left(2 r_{0}, 2 r_{0}+2\right)$ diagrams in which one pair of outer boundary points is connected to its nearest neighbor and all other strings are through strings, excluding the one in which -2 is connected to the neighboring boundary point with negative imaginary part. If the vector $w_{r_{0}}$ is normalized to be a unit vector, we get vectors whose matrix of inner products is the $\left(2 r_{0}+1\right) \times\left(2 r_{0}+1\right)$ matrix with $\delta$ on the diagonal, one on the first off-diagonals and 0 elsewhere. (Careless choice of how the inside annulus boundary is connected to the outside will lead to powers of $\omega$ on the off-diagonal but they can be removed by renormalizing the vectors one after another.) The determinant of this matrix is well known to be $\left[2 r_{0}+2\right]=[a+1]$, given in $(5-1)$, and hence negative. This is impossible in a Hilbert space. 


\section{Figure 13}

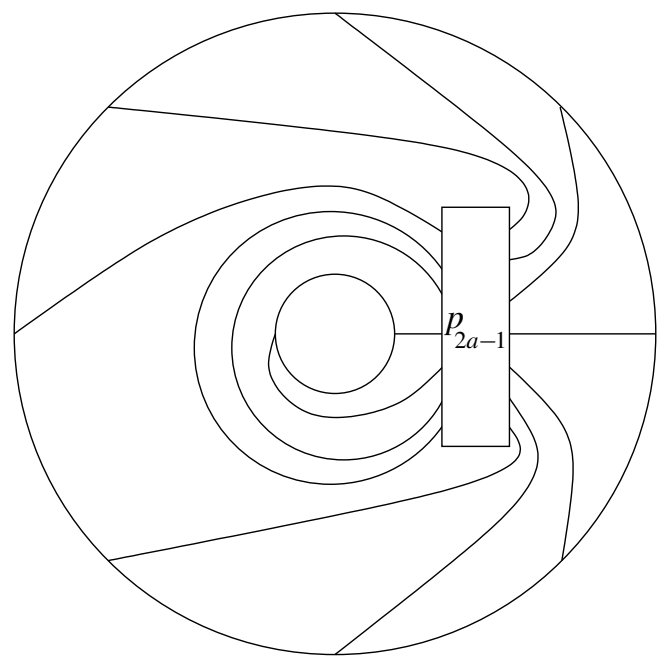

Case (ii) $a$ even so that $2 r_{0}=a-2$.

We will suppose that $k>0$. The case $k=0$ goes in exactly the same way but the diagrams need to be modified a little. We leave the details to the reader.

Here we will use the tangle encountered midway through the proof of Theorem 4.2. First let $\beta$ be the $(2 k, a)$ annular tangle with the $2 k$ internal boundary points connected to a JW idempotent on $a-1$ strings (the last one for which the inductive definition works) in a rectangle, $a-2 k-2$ boundary points of the rectangle connected pairwise by strings that go around the internal annulus boundary and one rectangle boundary point connected to -2 , as shown in Figure 13 for $a=8$ and $k=1$. The other $a-1$ rectangle boundary points are connected to the outer annulus boundary points.

The first thing we want to show is that $\beta\left(v_{\omega}\right)=0$. We do this by calculating $\left\langle\beta^{*} \beta\left(v_{\omega}\right), v_{\omega}\right\rangle$. Since $p_{a-1}$ is a projection, $\beta^{*} \beta$ is as in Figure 14 .

But in Figure 14 we see the JW idempotent with two boundary points capped off. In general this would be nonzero since the boundary points are not on the same side of the rectangle, but since this JW idempotent is the last one to exist, it spans the kernel of the natural inner product on TL diagrams so is invariant (at least up to a scalar) under the rotation. Thus if any two adjacent boundary points are connected the result is zero. (One can also show this by Wenzl's inductive formula.)

Thus $\beta\left(v_{\omega}\right)=0$.

We will now derive a contradiction by showing that the inner product of $\beta\left(v_{\omega}\right)$ with another vector is nonzero. This vector will be obtained from applying a $(2 k, a)$ tangle called $\gamma$ to $v_{\omega}$ where $\gamma$ is obtained from the $(2 k, a-2)$ tangle $\alpha_{a-2}$ of 


\section{Figure 14}

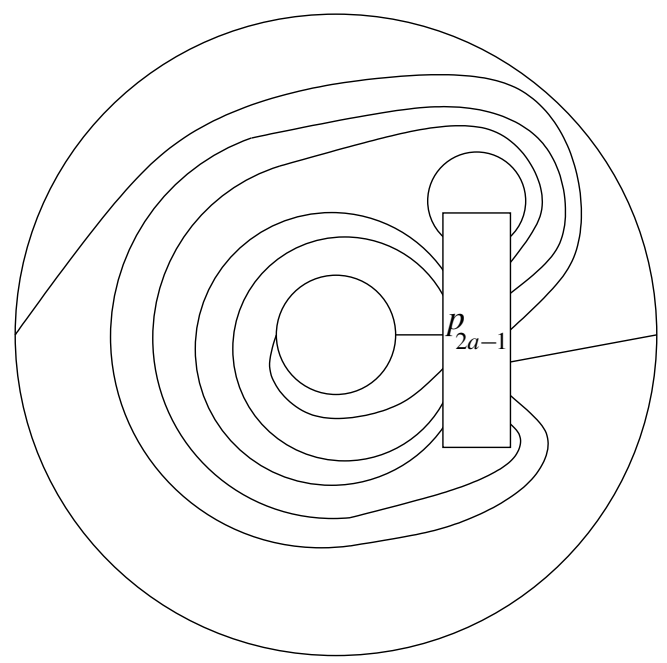

Figure 15

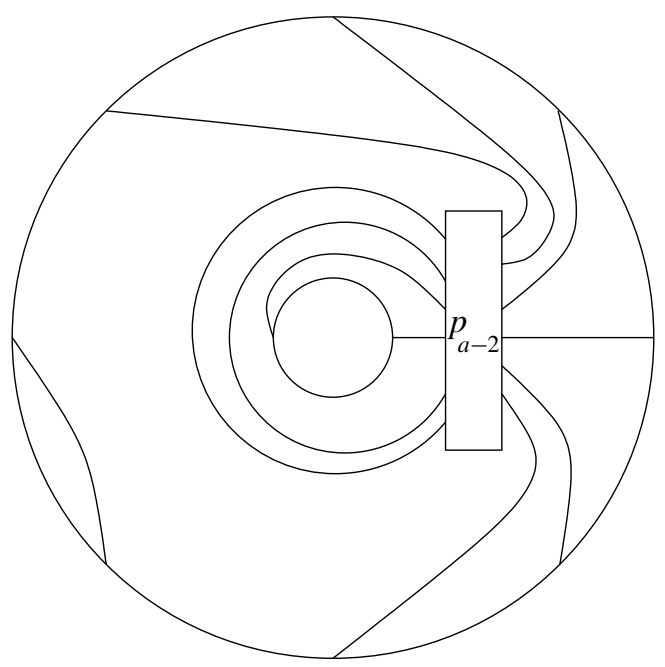

Definition 4.1 by connecting -2 to $-2 e^{(a+1) \pi i / a}$ and the other outer boundary points to the boundary points of the rectangle, as indicated in Figure 15.

To calculate the inner product $\left\langle\beta\left(v_{\omega}\right), \gamma\left(v_{\omega}\right)\right\rangle$ we use the tangle $\gamma^{*} \beta$, which we have drawn in Figure 16.

As in the proof of Theorem 4.2, there are only two diagrams that can be put in the rectangle which give nonzero contributions- those in which the boundary points connected to the first and last internal annular boundary points are connected to their neighbors (which are not connected to the inner annulus boundary). The coefficients of these diagrams are (from Figure 7)

$$
\frac{[a / 2-k]}{[a-1]} \text { and } \frac{[a / 2+k]}{[a-1]} \text {. }
$$




\section{Figure 16}

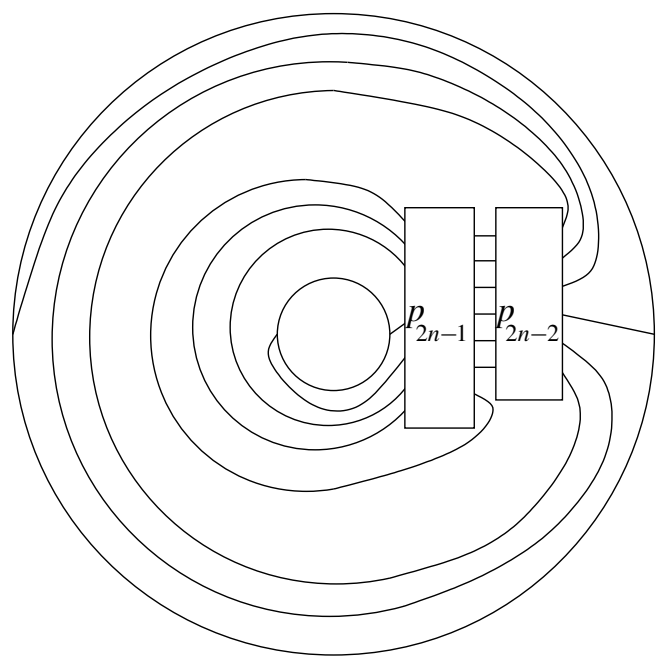

But these are both equal to

$$
\frac{\cos k \pi / a}{\sin (a-1) \pi / a},
$$

which is nonzero. On the other hand, the two resulting tangles are ( $\delta$ times) $\alpha_{a-2}^{*} \alpha_{a-2} \rho$ and $\alpha_{a-2}^{*} \alpha_{a-2}$. Since $\omega \neq-1$ (by the assumption on $\theta$ ), the sum of these two tangles applied to $v_{\omega}$ is nonzero. Hence, by Theorem 4.2, $\left\langle\beta\left(v_{\omega}\right), \gamma\left(v_{\omega}\right)\right\rangle$ is nonzero, a contradiction.

We point out two corollaries of Theorem 5.1. The first is immediate but somehow surprising.

Corollary 5.2. Let $U$ be a Hilbert space representation of AffTL. Then none of the rotations $\rho_{t}$ for $t \geq 1$ acts by the identity.

Proof. One may reduce to the irreducible case by using a maximal abelian subalgebra in the commutant of the algebra acting on the lowest weight space. Then the result follows from Theorem 5.1.

In [Jones 2001] we studied representations of the quotient AnnTL of our AffTL in which the rotations by $2 \pi, \rho_{t}^{t}$, act by the identity. We know that any Hilbert space representation of AnnTL will give one of AffTL, so we now identify those ones allowed by Theorem 5.1.

Corollary 5.3. Let $U$ be an irreducible Hilbert space representation of AffTL with chirality $\omega$ and lowest weight $k>0$. Then $u$ passes to AnnTL if and only if there is an integer $b$ such that $a=r k / b$. 
Proof. The main thing is that $\rho_{k}^{k}=1$ on $\boldsymbol{U}_{k, \pm}$ implies that $\rho_{n}^{n}=1$ on $\boldsymbol{U}_{n, \pm}$ for all $n \geq k$. This is because $U$ is a quotient of $V^{k, \omega}$ and it is clear that $\rho_{n}^{n} \alpha=\alpha \rho_{k}^{k}$ for any $\alpha \in \operatorname{AffTL}_{(k \operatorname{sgn}),(n, \pm s g n)}$.

So whenever $\omega$ is a $k$-th root of unity, $U$ passes to AnnTL. This is the condition $a=r k / b$ combined with the conclusion of Theorem 5.1.

\section{Construction of the allowed Hilbert space representations}

In this section we will undertake the most difficult part of this paper, namely the explicit construction of a representation $\mathscr{V}^{k, \omega}$ for each pair $(k, \omega)$ allowed by Theorem 5.1.

Theorem 6.1. Let $(k, \omega)$ be a pair where $k$ is a nonnegative integer and $\omega$ is a complex number $($ or $\omega=(-1, \pm)$, if $k=0)$. Then ${ }^{k}{ }^{k} \omega$ exists if

(i) $\delta \geq 2$ and either $k=0$ and $0 \leq \mu \leq \delta$,

or $k>0$ and $|\omega|=1$, or

(ii) $\delta=2 \cos \pi / a$ for $a=3,4,5, \ldots$, and $\omega=q^{ \pm 2 r}$ for some integer $r$ with $k<r \leq a / 2$ (where by -1 we mean $(-1, \pm$ ) if $k=0$ ).

Proof. As observed in Section 3, it suffices to show that the sesquilinear forms $\langle\cdot, \cdot\rangle$ of Definition 3.8 are positive semidefinite on $V^{k, \omega}$. The method, as in [Jones 2001], where it is done for the generic case, is to inductively decompose the representation $V_{(n, \pm)}^{k, \omega}$ with respect to a large ordinary TL subalgebra, which we will soon define.

First let us completely handle the case $q=1$ (so that $\delta=2$ ). When $\omega \neq 1$, we can inductively decompose the representation as is done for $\delta>2$ in [Jones 2001], and then confirm positive definiteness on the span of the vector $\omega_{n}$ by using Theorem 4.2. The case $\omega=1$ is quite different, as the form is only positive semidefinite and we must identify the kernel. In this case, it follows from the same theorem that $C_{n}=0$ for all $n \geq k+1$, so that in fact $\mathcal{V}^{k, \omega}$ is generated by $V_{k}^{k, 1}$.

In the case $\delta<2$, the large ordinary TL subalgebra will eventually fail to be semisimple. Semisimplicity is so important to our analysis - it allows us to use the inductive technique of [Jones 2001] — that we begin our proof by taking a quotient of $V^{k, \omega}$ on which, by Theorem 2.1, the action of TL passes to the $C^{*}$ quotient, $\mathscr{T} \mathscr{L}_{n}$.

It will be convenient to consider only the spaces $V_{n,+}^{k, \omega}$. The rotation provides an isometry between this and $V_{n,-}^{k, \omega}$ in all but a special case when $k=0$, where the situation is clear.

Thus let $\delta=2 \cos \pi / a$ for $a=3,4,5, \ldots$ and let $\omega$ and $k$ be as in the statement of the theorem. Define the subspace $\mathscr{G} W_{n, \pm}^{k, \omega}$ of $V_{n, \pm}^{k, \omega}$ to be the span of the image of $v_{\omega}$ under annular diagrams containing a rectangle labeled by the JW idempotent $p_{a-1}$ 
(see Remark 3.3). The subspaces $\mathscr{E} W_{n, \pm}^{k, \omega}$, as $n$ varies, are clearly invariant under the action of AffTL. We claim that they are in the kernel of $\langle\cdot, \cdot\rangle$. To see this, it suffices to show that an annular $(k, k)$ diagram containing the JW idempotent $p_{a-1}$ in a rectangle is zero modulo diagrams with fewer than $k$ through strings. Let $\alpha$ be such a diagram. We shall show that it has the same form as the element $\alpha_{n}^{*} \alpha_{n}$ of Theorem 4.2 and then apply the Graham-Lehrer formula.

If $\alpha$ had a string connecting the inside annulus boundary to the outside, then, cutting along that string, the rectangle would lie in a disc (after isotopy) with $4 k-2$ boundary points. The rectangle itself has $2 a-2$ boundary points and $k<a / 2$ so some boundary point on the rectangle must be connected to its nearest neighbor, which gives zero. So all of the strings from the inner annulus boundary are connected to the rectangle as are all those from the exterior annulus boundary. In fact $k \leq a / 2-1$ so that $4 k \leq 2 a-4$ so there are boundary points on the rectangle that are not connected to the annulus boundary. If these points were not connected symmetrically around the interior of the annulus there would be a rectangle boundary point connected to its nearest neighbor.

Let us first treat the case where $a$ is odd so the rectangle has an even number of boundary points at the top and at the bottom. Since the JW idempotent is rotationally invariant (up to a scalar, as observed in the proof of Theorem 5.1), and since $v_{\omega}$ is invariant up to a scalar, we may suppose that $\alpha$ is as in Figure 2 (which illustrates the case $a=9, n=3)$. Thus $\left\langle\alpha\left(v_{\omega}\right), v_{\omega}\right\rangle$ is equal to $C_{(a-1) / 2}$ and thus, by Theorem 4.2, proportional to $C_{r}$ since $r \leq(a-1) / 2$. But, again by the same theorem, $C_{r}=0$ since $\omega=q^{2 r}$.

Now turn to the case where $a$ is even. Then by rotational invariance as above we may suppose that $\alpha$ is as in Figure 16.

First suppose $r=a / 2$ so $\omega=-1$. Then by the argument after Figure 16 we get that $\left\langle\alpha\left(v_{\omega}\right), v_{\omega}\right\rangle=0$ precisely because $\omega=-1$ (which is the value it did not take there). The case $k=0$ is slightly different. In this case there is only one diagram, shown in Figure 17, that can be put into the rectangle to give a nonzero contribution, and that results in a homologically nontrivial circle surrounding the inner annulus boundary. This will give zero for $\left\langle\alpha\left(v_{\omega}\right), v_{\omega}\right\rangle$.

Finally if $r<a / 2$ we also get the situation of Figure 16, and again by the argument after that figure, we see that $\left\langle\alpha\left(v_{\omega}\right), v_{\omega}\right\rangle$ is proportional to $C_{a / 2-1}$. By Theorem 4.2, $C_{a / 2-1}$ is proportional to $C_{r}$, but as $\omega=q^{2 r}$ and $k<r$, Theorem 4.2 applies again to give $C_{r}=0$.

At this stage we have shown that in all cases allowed by the theorem, $\mathscr{E} W$ is contained in the kernel of $\langle\cdot, \cdot\rangle$, so that $\langle\cdot, \cdot\rangle$ defines an invariant form on the quotient AffTL-module $\Theta^{k, \omega}=V^{k, \omega} / \mathscr{f} W^{k, \omega}$. Positive semidefiniteness of $\langle\cdot, \cdot\rangle$ on $\Theta_{k, \omega}$ would therefore imply it on $V^{k, \omega}$. We will establish this positivity by restricting to a large ordinary TL algebra of $\operatorname{AffTL}_{(n,+),(n,+)}$ for $n \geq 1$. 


\section{Figure 17}

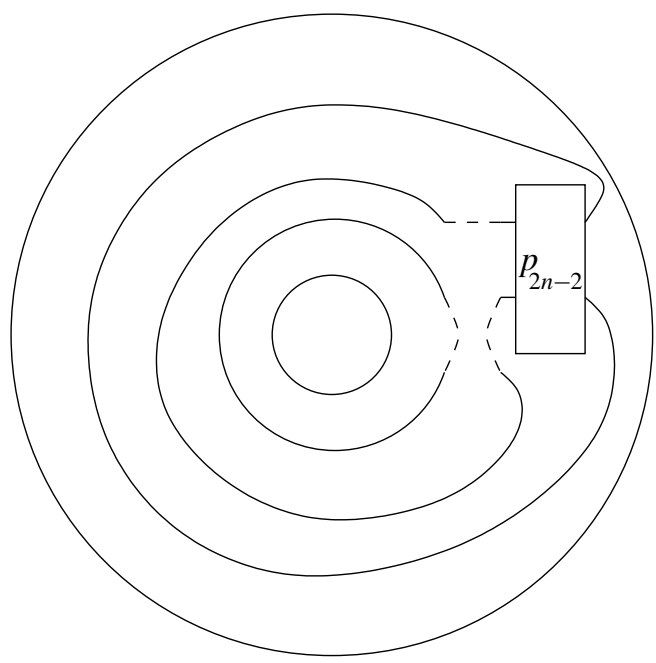

The intersection of the annulus $A$ with the complement of the wedge

$$
\left\{r e^{i \theta} \in \mathbb{C}:\left(1+\frac{1}{n+2}\right) \pi<\theta<\left(1+\frac{1}{n+1}\right) \pi\right\}
$$

is isotopic to a rectangle with $2 n$ marked points on the top and on the bottom. Thus the subalgebra of $\operatorname{AffTL}_{(n,+),(n,+)}$ spanned by isotopy classes of tangles that lie outside the wedge is isomorphic to the Temperley-Lieb algebra $\mathrm{TL}_{2 n}$. We will call it $t \ell_{2 n}$. Define $F_{n} \in \operatorname{AffTL}_{(n,+),(n,+)}$ to be the unique diagram in $t \ell_{2 n}$ with $2 n-2$ through strings and -1 connected to $e^{i(1-1 / n) \pi}$. One may jiggle the boundary points to exhibit an isomorphism between $F_{n} t \ell_{2 n} F_{n}$ and $t \ell_{2(n-1)}$, which makes $F_{n} V_{(n,+)}^{k, \omega}$ into a $t \ell_{2(n-1)}$-module isomorphic to $V_{(n-1,+)}^{k, \omega}$.

All this is as in [Jones 2001]. Moreover, these isomorphisms take the subspace $\mathscr{E} W_{(n,+)}^{k, \omega}$ to the subspace $\mathscr{E} W_{(n-1,+)}^{k, \omega}$ and the ideal $\mathscr{I}_{2 n}$ generated in $t \ell_{2 n}$ by $p_{a-1}$ (which we take to be zero when $2 n<a-1$ ) onto $\mathscr{I}_{2 n-2}$. It is obvious that the ideal $\mathscr{I}_{2 n}$ preserves $\mathscr{E} W_{(n,+)}^{k, \omega}$ so that the quotients $\mathscr{X}_{n}^{k, \omega}=V_{(n,+)}^{k, \omega} / \mathscr{\mathscr { S }} W_{(n,+)}^{k, \omega}$ become modules over the quotients $\mathscr{T} \ell_{2 n}=t \ell_{2 n} / \mathscr{I}_{2 n}$. But by Theorem 2.1, $\mathscr{T} \ell_{2 n}$ is a finite-dimensional $C^{*}$-algebra (with irreducible representations as described in Section 2). Further, by using the isomorphisms established above, we see that for every value of $t, F_{n} \mathcal{W}_{2 n}^{t}$ is isomorphic to $\mathcal{W}_{2 n-2}^{t}$ as a $\mathcal{T} \ell_{2 n-2}$-module.

Thus $\mathscr{X}_{n}^{k, \omega}$ as a $\mathscr{T} \ell_{2 n}$-module is a direct sum of as many copies of each $\mathscr{W}_{2 n}^{t}$ as $\mathscr{2}_{n-1}^{k, \omega}$ is of $\mathcal{W}_{2 n-2}^{t}$, plus a certain number of copies of the trivial representation if $2 n<a-1$ (and none if $2 n \geq a-1$ ). The form $\langle\cdot, \cdot\rangle$ is invariant under $t \ell$ and there is only one such form (up to a scalar) on $W$, so positive semidefiniteness on the span of the nontrivial representations follows by induction as soon as it is established on the trivial ones as they appear. (The statement for the generic case is [Jones 
2001, Proposition 4.10], the proof of which goes through as long as $2 n \leq a-1$.) But the JW idempotent $p_{2 n}$ projects onto the trivial representation for $2 n<a-1$, and annihilates all vectors in $V_{n,+}^{k, \omega}$ except for

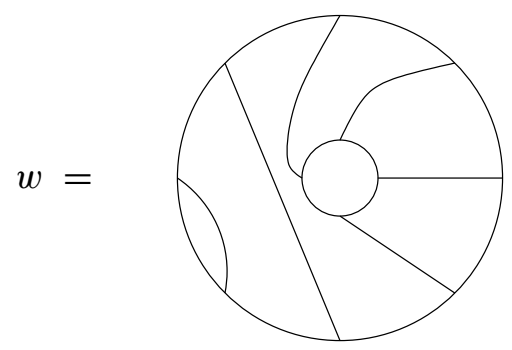

where all strings that connect the outside boundary to itself go through the wedge defined above.

Thus there is exactly one copy of the trivial representation for $2 n<a-1$ and none for $2 n \geq a-1$. Inspection of the preceding figure shows that $p_{2 n}(w)=\alpha_{n}\left(v_{\omega}\right)$ so that positive semidefiniteness of $\langle\cdot, \cdot\rangle$ follows from $C_{n} \geq 0$. This follows from Theorem 4.2 and the choice of $k, \omega$ and $\delta$, which force $C_{n}$ to be zero before it has a chance to be negative.

We see in the proof of the theorem that we have in fact determined the structure of the Hilbert space representations as modules over the large Temperley-Lieb subalgebra. In fact the $\mathcal{V}_{n,+}^{k, \omega}$ become modules over the TL algebroid discussed in Remark 2.4. The ordinary TL algebroid is spanned by all diagrams in $\operatorname{AffTL}_{(m,+),(n,+)}$ that do not intersect the wedge

$$
\left\{r e^{i \theta} \in \mathbb{C}:\left(1+\frac{1}{r+2}\right) \pi<\theta<\left(1+\frac{1}{r+1}\right) \pi\right\},
$$

where $r=\max (m, n)$. The Hilbert space representations of this algebroid are precisely the modules $\mathscr{W}^{t}$.

Scholium 6.2. Suppose $\omega$ and $k$ satisfy the conditions of Theorem 6.1. For any $n \geq k$, as a module over the ordinary TL algebroid, $\mathscr{V}_{(n,+)}^{k, \omega}$ is

(i) $w_{2 k}^{2 k} \quad$ if $\delta=2$ and $q=1$,

(ii) $\bigoplus_{j=k}^{r-1} W_{2 n}^{2 j} \quad$ if $k \geq 0$, or $k=0$ and $\omega \neq(-1, \pm)$.

(iii) $\bigoplus \mathcal{W}_{2 n}^{2 j} \quad$ if $k=0$ and $\omega=(-1,+)$, $0 \leq j \leq m$

(iv) $\bigoplus \mathcal{W}_{2 n}^{2 j} \quad$ if $k=0$ and $\omega=(-1,-)$, 
where in (iii) and (iv) $m=a / 2-1$. (Note that for these values of $\omega, a$ is guaranteed to be even, so $m$ is indeed an integer.)

Proof. The first statement follows immediately from the remarks at the beginning of this section.

Item (ii) follows from the inductive procedure used to decompose $\mathcal{V}_{(n,+)}^{k, \omega}$ as a module over the ordinary TL algebroid, which relies on the following two facts from [Jones 2001]. First, when $2 n \leq a-1$ and $0 \leq j<n$, a representation $\pi$ of $\mathcal{T} \ell_{2 n}$ contains a copy of $\mathscr{W}_{2 m}^{2 j}$ if and only if $\pi$ restricted to $E_{2 n-1}\left(\mathcal{T} \ell_{2 n}\right) E_{2 n-1}$ contains a copy of $W_{2 n-2}^{2 j}$ : this is Proposition 4.10 of [Jones 2001] (with a weakened assumption on the genericity of the index, which does not affect the proof). Second, Theorem B1 of the same paper says that if $\mathscr{V}_{(m,+)}^{k, \omega}$ has dimension $\left(\begin{array}{c}2 m \\ m-k\end{array}\right)-1$ then for all $n \geq m$, as a $\mathcal{T} \ell_{2 n}$ module, $\mathscr{V}_{(n,+)}^{k, \omega}$ is isomorphic to $\bigoplus_{j=k}^{m-1} \mathcal{W}_{2 n}^{2 j}$. (Note that the hypothesis on the dimension is satisfied since $r \leq a / 2$.)

Parts (iii) and (iv) are proved by introducing a second copy of the ordinary Temperley-Lieb algebroid, $\widetilde{\mathcal{T} \ell_{2 n}}$, and arguing that (for $\left.n>1\right) \mathscr{W}_{2 j}^{2 n}$ is present in $\mathscr{V}_{(n,+)}^{k, \omega}$ as a $\mathscr{T} \ell_{2 n}$-module when $j$ is odd and as a $\widetilde{\mathscr{T} \ell_{2 n}}$ module when $j$ is even. The induction is as in Theorem 5.23 of [Jones 2001]. An adjustment of the dimension hypothesis of Theorem B.1 of the same paper can be made for this case so that that proof guarantees the form of the $\mathcal{V}_{(n,+)}^{k, \omega}$ when $n \geq a$.

Corollary 6.3. The dimension of $\mathcal{q}^{k, \omega}$ is

(i) $z^{k} \frac{C(z)^{2 k}-z C(z)^{2 k+2}}{\sqrt{1-4 z}}$ if $\delta=2$ and $q=1$,

(ii) $\frac{1}{Q_{a}(z)} \sum_{j=k}^{r-1} z^{j} Q_{a-2 j-1}(z) \quad$ if $\omega=q^{ \pm 2 r}$,

(iii) $\frac{1}{2}+\frac{1}{2 Q_{a}(z)} \sum_{j=0}^{a / 2-1} z^{j} Q_{a-2 j-1}(z)$ if $k=0, \omega=(-1,+)$,

(iv) $-\frac{1}{2}+\frac{1}{2 Q_{a}(z)} \sum_{j=0}^{a / 2-1} z^{j} Q_{a-2 j-1}(z)$ if $k=0, \omega=(-1,-)$.

Proof. To prove part (i), note that $\operatorname{dim} W_{n}^{t}=\left(\begin{array}{c}2 n \\ n-t\end{array}\right)-\left(\begin{array}{c}2 n \\ n-t-1\end{array}\right)$, so by [Graham et al. 1994, page 203], we obtain the result. Part (ii) follows directly from Theorem 2.3 and the scholium. For (iii) and (iv) use the fact that $\mathscr{q}^{k, \omega}$ is isomorphic to both

$$
\bigoplus_{\substack{1 \leq j \leq m \\ j \in 2 \mathbb{N}}} W_{2 n}^{2 j} \text { and } \bigoplus_{\substack{1 \leq j \leq m \\ j+1 \in 2 \mathbb{N}}} W_{2 n}^{2 j},
$$

so these must have equal dimension. 


\title{
References
}

[Goodman et al. 1989] F. M. Goodman, P. de la Harpe, and V. F. R. Jones, Coxeter graphs and towers of algebras, Mathematical Sciences Research Institute Publications 14, Springer, New York, 1989. MR 91c:46082 Zbl 0698.46050

[Graham and Lehrer 1998] J. J. Graham and G. I. Lehrer, "The representation theory of affine Temperley-Lieb algebras”, Enseign. Math. (2) 44:3-4 (1998), 173-218. MR 99i:20019 Zbl 0964. 20002

[Graham et al. 1994] R. L. Graham, D. E. Knuth, and O. Patashnik, Concrete mathematics, 2nd ed., Addison-Wesley, Reading, MA, 1994. A foundation for computer science. MR 97d:68003 Zbl 0836.00001

[Jones 1983] V. F. R. Jones, “Index for subfactors”, Invent. Math. 72:1 (1983), 1-25. MR 84d:46097 Zbl 0508.46040

[Jones 1994] V. F. R. Jones, "A quotient of the affine Hecke algebra in the Brauer algebra”, Enseign. Math. (2) 40:3-4 (1994), 313-344. MR 95j:20038 Zbl 0852.20035

[Jones 1999] V. F. R. Jones, "Planar algebras, I", preprint, 1999. To appear in New Zealand J. Math. math.QA/9909027

[Jones 2001] V. F. R. Jones, “The annular structure of subfactors”, pp. 401-463 in Essays on geometry and related topics, edited by E. Ghys et al., Monogr. Enseign. Math. 38, Enseignement Math., Geneva, 2001. MR 2003j:46094 Zbl 1019.46036

[Kauffman 1987] L. H. Kauffman, "State models and the Jones polynomial”, Topology 26:3 (1987), 395-407. MR 88f:57006 Zbl 0622.57004

[Wenzl 1987] H. Wenzl, "On sequences of projections", C. R. Math. Rep. Acad. Sci. Canada 9:1 (1987), 5-9. MR 88k:46070 Zbl 0622.47019

Received March 12, 2005.

VAUGHAN F. R. JONES

DEPARTMENT OF MATHEMATICS

UNIVERSITY OF CALIFORNIA, BERKELEY

BERKELEY, CA 94720-3840

UNITED STATES

vfr@math.berkeley.edu

http://math.berkeley.edu/ vfr/

\author{
SARAH A. REZNIKOFF \\ DEPARTMENT OF MATHEMATICS AND STATISTICS \\ UNIVERSITY OF VICTORIA \\ VICTORIA, BC V8W 3P4 \\ CANADA \\ sarah@math.uvic.ca \\ http://www.math.uvic.ca/ sarah
}

